\title{
British Thoracic Society Guideline for the investigation and management of malignant pleural mesothelioma
}

Ian Woolhouse, ${ }^{1}$ Lesley Bishop, ${ }^{2}$ Liz Darlison, ${ }^{3}$ Duneesha De Fonseka, ${ }^{4}$ Anthony Edey, ${ }^{5}$ John Edwards, ${ }^{6}$ Corinne Faivre-Finn, ${ }^{7}$ Dean A Fennell, ${ }^{8}$ Steve Holmes, ${ }^{9}$ Keith M Kerr, ${ }_{1}^{10}$ Apostolos Nakas, ${ }^{11}$ Tim Peel, $^{12}$ Najib M Rahman, ${ }^{13}$ Mark Slade, ${ }^{14}$ Jeremy Steele, ${ }^{15}$ Selina Tsim, ${ }^{16}$ Nick A Maskell ${ }^{17}$

- Additional material is published online only. To view, please visit the journal online (http://dx.doi.org/10.1136/ thoraxinl-2017-211321).

For numbered affiliations see end of article.

\section{Correspondence to} Professor Nick A Maskell, Academic Respiratory Unit, Bristol Medical School, University of Bristol, BS10 5NB, UK; nick.maskell@bristol.ac.uk
Check for updates

To cite: Woolhouse l, et al. Thorax 2018;73:i1-i30.

\section{SUMMARY OF RECOMMENDATIONS AND GOOD PRACTICE POINTS \\ Section 3: Clinical features which predict the presence of mesothelioma \\ Recommendations}

- Do not rule out a diagnosis of malignant pleural mesothelioma (MPM) on the basis of symptoms and examination findings alone. Grade D.

- Offer an urgent chest X-ray to patients with symptoms and signs as outlined in the National Institute for Health and Care Excellence (NICE) NG12. Grade D.

- Refer all patients with a chest X-ray suggestive of MPM urgently (via the 2-week wait suspected cancer pathway in England and Wales). Consider referral for further investigation in patients with persistent symptoms and history of asbestos exposure despite normal chest X-ray. Grade D.

- A thorough occupational history should be taken to cover all occupations throughout life. It is important to elicit para exposure by exploring details of relative and/or partner occupations. Grade D.

\section{Section 4: Staging systems}

\section{Recommendation}

- Record staging of MPM according to the version 8 of the International Association for the Study of Lung Cancer (IASLC) staging proposals. Grade D.

Section 5: Imaging modalities for diagnosing and staging

Recommendations

- Offer CT thorax with contrast (optimised for pleural evaluation) as the initial cross-sectional imaging modality in the evaluation of patients with suspected MPM. Grade D.

- Use of positron emission tomography (PET)-CT for aiding diagnosis of MPM is not recommended in patients who have had prior talc pleurodesis and caution should be employed in populations with a high prevalence of TB. Grade D.

- In patients where differentiating $\mathrm{T}$ stage will change management, consider MRI. Grade D.

- In patients where excluding distant metastases will change management, offer PET-CT. Grade D.
Section 6: Pathological diagnosis

\section{Recommendations}

- Immunohistochemistry (IHC) is recommended for the differential diagnosis of MPM in both biopsy and cytology-type specimens. Grade D.

- A combination of at least two positive mesothelial (calretinin, cytokeratin $5 / 6$, Wilms tumour $1, \mathrm{D}-240)$ and at least two negative adenocarcinoma immunohistochemical markers (TTF1, CEA, Ber-EP4) should be used in the differential diagnosis of MPM. (Markers listed in likely order of value). Grade D.

- Do not rely on cytology alone to make a diagnosis of MPM unless biopsy is not possible or not required to determine treatment due to patient's wishes or poor performance status (PS). Grade D.

- Pathologists should report the histological subtype of MPM in all cases. Grade D.

\section{Good practice points}

$\checkmark$ Biopsies from patients with suspected MPM should be reviewed by a pathologist experienced in the diagnosis of MPM and a second opinion should be sought if there is uncertainty over the diagnosis.

Section 7: Use of biomarkers

Recommendations

- Do not offer biomarkers in isolation as a diagnostic test in MPM. Grade B.

- Consider biomarker testing in patients with suspicious cytology who are not fit enough for more invasive diagnostic tests. Grade B.

- Do not routinely offer biomarker testing to predict treatment response or survival. Grade B.

- Do not offer biomarker testing to screen for MPM. Grade C.

\section{Research recommendation}

Further research is required to identify biomarkers that reliably predict treatment response within clinical practice.

Section 8: Factors determining prognosis and timing of treatment

Recommendations

- Consider calculating a prognostic score in patients with MPM at diagnosis. Grade D. 
- Prognostic scores can provide useful survival information for patients and doctors, but should not be used in treatment decision-making. Grade D.

- When calculating a prognostic score use one of the following:

a. The European Organisation for the Research and Treatment of Cancer (EORTC) prognostic score;

b. The CALGB score;

c. The modified Glasgow prognostic score (mGPS);

d. The LENT score if a pleural effusion is present;

e. The decision tree analysis.

The decision tree analysis scoring systems is likely to be the most useful in routine clinical practice. Grade D.

\section{Section 9: Pleural fluid management Recommendations}

- Offer either talc (via slurry or poudrage) or indwelling pleural catheters for symptomatic pleural effusion in MPM, informed by patient choice. Grade A.

- Talc slurry or thoracoscopic talc poudrage pleurodesis should be offered to patients with MPM in preference to a videoassisted thoracoscopic surgery partial pleurectomy (VATSPP) surgical approach for fluid control in MPM. Grade A.

\section{Section 10: The role of surgery \\ Recommendations}

- Do not offer VATS-PP over talc pleurodesis in MPM. Grade A.

- Do not offer Extra-Pleural Pneumonectomy (EPP) in MPM. Grade B.

- Do not offer extended pleurectomy decortication (EPD) outside of a clinical trial. Grade D.

\section{Research recommendation}

The role of VATS-PP and EPD in good prognosis patients should be examined further in clinical trials, which should include robust measurement of quality of life.

\section{Section 11: Systemic anticancer treatment Recommendations}

- Offer patients with MPM with good PS (WHO 0-1) firstline therapy with cisplatin and pemetrexed. Where licensed (not presently in the UK), bevacizumab should be added to this regime. Raltitrexed is an alternative to permetrexed. Grade A.

- Do not offer pemetrexed or vorinostat as second-line treatment for patients with MPM. Grade A.

\section{Good practice points}

$\checkmark \quad$ Where cisplatin is contraindicated, or has adverse risk, offer carboplatin in combination with pemetrexed.

$\checkmark \quad$ First-line clinical trials are an appropriate option for patients with good PS and are recommended above any other option for second-line treatment, providing the patient is of adequate PS.

\section{Research recommendations}

The role of immunotherapy in MPM should be further assessed in large phase III randomised controlled trials (RCTs).
Further randomised controlled trials of second-line therapy on MPM are required.

\section{Section 12: Radiotherapy}

\section{Recommendations}

- Do not offer prophylactic radiotherapy to chest wall procedure tracts. Grade A.

- Do not offer preoperative or postoperative radiotherapy in MPM. Grade A.

- Do not offer hemithorax radiotherapy for MPM. Grade D.

- Consider palliative radiotherapy for localised pain in MPM, where the pain distribution matches areas of underlying disease. Grade D.

\section{Research recommendations}

Prospective clinical trials of preoperative radiotherapy, postoperative radiotherapy after pleurectomy decortication and definitive radiotherapy after chemotherapy in MPM are required.

Further prospective randomised clinical trials are required to determine the role of radiotherapy for symptom control in MPM and the optimal dose fractionation.

\section{Section 13: Symptom control}

Good practice point

$\checkmark$ Symptoms in MPM should be managed as per current guidelines for cancer in general (table 18) and early involvement of palliative care specialists is recommended.

\section{Section 14: Care and management}

\section{Recommendations}

- Consider referring MPM cases to a regional mesothelioma MDT. Grade D.

- Offer accurate and understandable information to patients and carers about compensation for MPM. Grade D.

- Offer patients with MPM and their carers the opportunity to discuss concerns regarding their disease. Grade D.

- In patients with MPM where accurate determination of radiological progression is required, consider CT with modified Response Evaluation Criteria In Solid (mRECIST) measurement. Grade D.

\section{Good practice points}

$\checkmark$ All mesothelioma cases should be discussed in a timely fashion by a MDT that reviews a sufficient number of cases to maintain expertise and competence in the diagnosis and treatment of MPM.

$\checkmark$ The MDT membership should fulfil the requirements set by national cancer peer review (to include a named clinical nurse specialist for MPM).

$\checkmark \quad$ The MDT should maintain an up-to-date portfolio of mesothelioma trials and offer recruitment to all eligible patients.

$\checkmark$ A personalised care approach should be considered for each patient.

Patients should be offered 3-4 monthly follow-up appointments with an oncologist, respiratory physician or specialist nurse according to their current treatment plan. If patients wish to be seen less frequently, offer regular telephone follow-up with specialist nurse with an option to attend clinic in the event of clinical deterioration. 


\section{SECTION 1: INTRODUCTION}

\section{Aim of the guideline}

The key aim of this guideline is to provide detailed, evidencebased guidance for the investigation of suspected MPM and the subsequent care and management of individuals with proven MPM. MPM is a rare cancer where the malignancy affects the pleura, a thin membrane of lubricating cells that lines the lungs and chest wall. The focus of this guideline is MPM as it is far more common than mesothelioma occurring in the abdomen. The 2016 Mesothelioma Audit data reported that in the UK in 2014 pleural mesothelioma accounted for 2179 cases (97\%), with 70 peritoneal cases (approximately $3 \%$ ). ${ }^{1}$

In 2007, the British Thoracic Society (BTS) statement on mesothelioma was published in response to a request from the National Health Executive in England. ${ }^{2}$ The BTS has reviewed this statement and is of the opinion that the publication is no longer fit for purpose as an up-to-date reference guide for healthcare professionals. The 2007 statement did not attempt to provide a comprehensive review of all relevant published literature and since the publication of the statement the BTS has achieved NICE accreditation for its guideline production process. The Standards of Care Committee (SOCC) of the BTS established a guideline development working group in 2014, chaired by Professor Nick Maskell and Dr Ian Woolhouse.

The main cause of mesothelioma is breathing in asbestos dust-approximately $85 \%$ of all mesotheliomas in males are attributable to occupational asbestos exposures. The use of products containing asbestos was banned in the UK in 1999. The latency period between first exposure and development of the disease is very long, typically $30-40$ years.

Cases of mesothelioma were recorded systematically from the late 1960s. The incidence of mesothelioma has been increasing steadily since then, and current predictions suggest there will continue to be approximately 2500 deaths per year for the rest of this decade, before numbers begin to fall. (HSE http://www. hse.gov.uk/Statistics/causdis/mesothelioma/mesothelioma.pdf).

The largest dataset of MPM in the UK comes from the National Lung Cancer Audit report, which described 8740 cases seen in hospitals in England and Wales between 2008 and $2012 .^{3}$ Eighty-three per cent of patients were male and the median age at diagnosis was 73 years. Sixty-seven per cent of patients received active anticancer treatment (chemotherapy, radiotherapy and surgery) and overall median survival was 9.5 months, with 1 -year and 3-year survival rates of $41 \%$ and $12 \%$, respectively. The report identified significant variation in treatment and outcomes across the UK which further highlights the need for an evidence-based guideline to facilitate the highest standards of care for all patients with mesothelioma in the UK.

\section{Intended users of the guideline and target patient populations}

The guideline will be primarily of interest to healthcare professionals working within the National Health Service (NHS), but the aim is to make the guideline as applicable to international practice as possible so that it may be used across Europe and America as appropriate. Given the nature of MPM, the majority of the guideline will be relevant to secondary care-based specialists; however, symptom recognition, management and follow-up are all relevant to community-based specialties.

\section{Intended users}

- Primary care-general practitioners (GPs) and practice nurses;
- Hospital specialist teams in respiratory medicine, oncology, thoracic surgery and palliative care;

- Hospices/community teams;

- Specialist nurses (including lung cancer and palliative care);

- Radiologists;

- Pathologists.

\section{Areas covered by the guideline}

Inclusion

- The epidemiology and incidence of mesothelioma in the UK and worldwide;

- The preferred investigation pathway of suspected cases of MPM;

- Consider special situations including:

- Imaging;

- Histology/cytology;

- Frail patient not fit for invasive tests.

- Biomarkers;

- Role of mesothelioma MDTs;

- Outline best practice in oncological management:

- Role of chemotherapy;

- Place for radiotherapy;

- Role of surgery.

- Guidance on palliation in MPM;

- Guidance on providing patients with relevant disease-specific information, including medicolegal/compensation issues;

- Summary of future therapeutic agents that might be available within next 5 years;

- Summary of major MPM recommendations.

\section{Areas not covered by the guideline}

Non-pleural mesothelioma is excluded from this guideline.

\section{Limitations of the guideline}

Healthcare providers need to use clinical judgement, knowledge and expertise when deciding whether it is appropriate to apply recommendations for the management of patients. The recommendations cited here are a guide and may not be appropriate for use in all situations. The guidance provided does not override the responsibility of healthcare professionals to make decisions appropriate to the circumstances of each patient, in consultation with the patient and/or their guardian or carer.

\section{Members of the guideline development group}

The guideline development group (GDG) was chaired by two respiratory consultants-Dr Ian Woolhouse and Professor Nick Maskell. The GDG had a wide membership with representation from respiratory medicine, thoracic surgery, medical oncology, radiotherapy, pathology and primary care. A patient representative was on the group for the duration of the process. Those on the group were not required to be BTS members.

\section{Representation}

Professor Dean Fennell and Dr Jeremy Steel represented the Association of Cancer Physicians. Dr Anthony Edey represented the British Society of Thoracic Imaging. Professor Corinne Faivre-Finn represented the British Thoracic Oncology Group. Professor Keith Kerr represented the Royal College of Pathologists. Dr Ian Woolhouse represented the Royal College of Physicians. Mr John Edwards and $\mathrm{Mr}$ Apostolos Nakas represented the Society of Cardiothoracic Surgeons. Dr Corinne-Faivre-Finn and Dr Anthony Edey represented the Royal College of Radiologists. Dr Tim Peel 
represented the Association for Palliative Medicine. Dr Steve Holmes represented the Primary Care Respiratory Society UK. Ms Liz Darlison represented the Royal College of Nursing. Dr Graham Abbott, Mr Paul Astle and Mr John Gillies were the patient representatives on the group.

\section{SECTION 2: METHODOLOGY OF GUIDELINE PRODUCTION Establishment of guideline development group}

The GDG was convened in June 2014, with the first meeting taking place in October 2014. The full GDG met six times during the development of the guideline and kept in close contact by teleconference and email throughout the process. The BTS SOCC reviewed the draft guideline in November 2016. The draft guideline was made available online from 22 March 2017 until 24 April 2017 for public consultation and circulated to all relevant stakeholders. The BTS SOCC reviewed the revised draft in June 2017 and final SOCC approval was granted in September 2017.

\section{Methodology}

This guideline is based on the best available evidence and follows the NICE-accredited BTS guideline production process. The methodology used to write the guideline adheres strictly to the criteria as set by the Appraisal of Guidelines, Research and Evaluation (AGREE) II collaboration, which is available online www. agreetrust.org/resource-centre/agree-ii/. The BTS SOCC guideline production manual is available at: https://www.brit-thoracic. org.uk/guidelines-and-quality-standards/

\section{Summary of key questions and literature search}

Clinical questions were gathered in the Patient, Intervention, Comparison, Outcome and Time (PICOT) format. The key questions are summarised below.

- Which clinical features predict the presence of MPM?

- In patients with suspected MPM (post chest X-ray), which imaging modality is best for diagnosis/staging and what technical factors are important?

- Should biomarkers (serum/fluid) be measured in MPM?

- Is there a staging system for MPM that determines management and predicts outcome?

- What factors determine prognosis and timing of treatment in MPM?

- What are the appropriate cytopathological approaches which allow diagnosis and subtyping of MPM?

- Is the care of patients with suspected/proven MPM improved by discussion at a specialist MDT?

- Where histological confirmation is either not possible or not definite, what are criteria for a clinical diagnosis of MPM?

- What is the optimum strategy for the management of pleural fluid in MPM?

- Is there a role for surgery in the management and treatment of patients with MPM?

- Is there a role for systemic anticancer treatment in MPM?

- Is there a role for radiotherapy in MPM?

- What treatment/interventions are effective for symptom control in MPM?

- What are the nursing care and information needs for patients with suspected and proven MPM?

- What is the most effective follow-up strategy of patients with MPM?

The PICOT framework was used to define the scope of the guideline and formed the basis of the literature search. The literature search was conducted in December 2014 by York University.
Systematic electronic database searches were conducted in order to identify all papers which may potentially be included in the guideline. For each question, the following databases were searched: Cochrane Database of Systematic Reviews (CDSR), Database of Abstracts of Reviews of Effects (DARE), Health Technology Assessment Database (HTA), Cochrane Central Register of Controlled Trials (CENTRAL), MEDLINE and MEDLINE In-Process, EMBASE and PUBMED (see online supplementary appendix 1).

The search was limited to papers published in English. The searches identified a total of 6173 abstracts. The full list of abstracts was retained and is kept in an archive. A second search was completed in July 2016 to search for relevant papers published between 2014 and 2016, yielding a further 1038 potentially relevant references. Additional references were included from personal collections as appropriate.

\section{Appraisal of the evidence}

An initial screen was completed to remove letters, conference papers and news articles. Dr Woolhouse and Professor Maskell read the remaining abstracts (5129), marked those considered relevant to the scope of the guideline and allocated each relevant abstract to a clinical question(s). 950 abstracts were allocated to clinical question(s). For the second search, the initial screen reduced the abstracts to 582 . These were all read by Dr Woolhouse and Professor Maskell and 44 were allocated to clinical question(s). GDG members were allocated to work on the questions in small groups.

Each abstract was read and at least two members agreed whether the paper was relevant to the particular guideline section. Papers were excluded if the following applied:

- If the paper did not answer the clinical question concerned.

- If it was a case report of fewer than 20 patients; however, this was not an absolute cut-off. Professional judgement was applied and some smaller case reports were considered, and indeed some case reports of more than 20 patients were excluded.

- If the language of the full paper was not English.

Full papers were obtained for all relevant, or possibly relevant, abstracts.

At least two members of each small group independently appraised each paper using the SIGN critical appraisal checklists. An evidence level was assigned to each study using the SIGN methodology (table 1 and 2).

\begin{tabular}{ll}
\hline Table 1 SIGN levels of evidence \\
\hline $1++$ & $\begin{array}{l}\text { High-quality meta-analyses, systematic reviews of randomised controlled } \\
\text { trials (RCTs), or RCTs with a very low risk of bias }\end{array}$ \\
\hline $1+$ & $\begin{array}{l}\text { Well-conducted meta-analyses, systematic reviews, or RCTs with a low risk } \\
\text { of bias }\end{array}$ \\
$1-$ & Meta-analyses, systematic reviews or RCTs with a high risk of bias \\
$2++$ & $\begin{array}{l}\text { High-quality systematic reviews of case-control or cohort or studies } \\
\text { High-quality case-control or cohort studies with a very low risk of } \\
\text { confounding or bias and a high probability that the relationship is causal }\end{array}$ \\
$2+$ & $\begin{array}{l}\text { Well-conducted case-control or cohort studies with a low risk of confounding } \\
\text { or bias and a moderate probability that the relationship is causal }\end{array}$ \\
$2-$ & $\begin{array}{l}\text { Case-control or cohort studies with a high risk of confounding or bias and a } \\
\text { significant risk that the relationship is not causal }\end{array}$ \\
3 & $\begin{array}{l}\text { Non-analytic studies, eg, case reports, case series } \\
4\end{array}$ \\
\hline
\end{tabular}

\section{Table 1 SIGN levels of evidence}

$1++\quad$ High-quality meta-analyses, systematic reviews of randomised controlled

Woolhouse I, et al. Thorax 2018;73:11-i30. doi:10.1136/thoraxjnl-2017-211321 


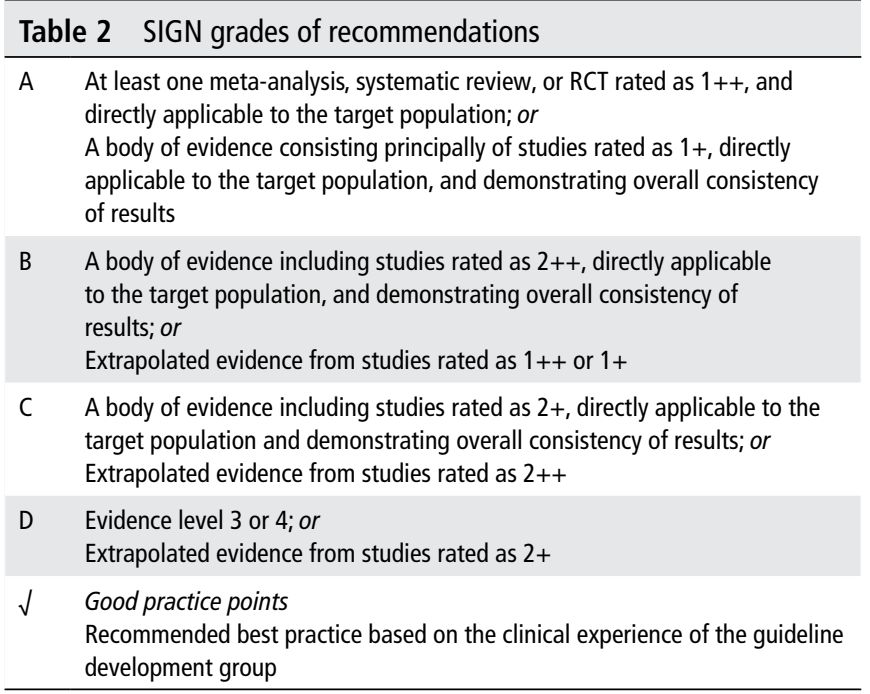

Each relevant paper was read in full by at least two members of the GDG and an evidence table entry was completed for each paper used to support a recommendation/good practice point. The full GDG reviewed each section during the regular meetings and consensus was reached. Evidence tables are available in the online supplementary appendix 2 .

From the outset, it was acknowledged that there would be little high-quality evidence for some of the clinical questions identified. In this instance, low-grade evidence was considered, along with expert opinion via consensus at the meetings.

The following parameters were used by the GDG to appraise the evidence:

- How applicable the obtained evidence was in making recommendations for the defined target audience of this guideline.

- Whether the evidence was generalisable and relevant to the target population for the guideline.

- Whether there was a clear consistency in the evidence obtained to support recommendations.

- What the implications of recommendations would be on clinical practice in terms of resources and skilled expertise.

Cost-effectiveness was not considered in detail as in-depth economic analysis of recommendations falls outside of the BTS guideline production process. However, the GDG were asked to be mindful of any barriers to implementing the recommendations and good practice points (GPPs).

Recommendations were graded from A to D as indicated by the strength of the evidence as shown in table 2 . In line with SIGN guidance, 'minus' evidence was considered where necessary, but only in such instances when there were no published 'plus' papers. In this context, any recommendation based on this evidence was made Grade D. GPPs were included where research evidence was lacking, but the GDG felt it was important to highlight practical points which could improve the care of patients. Research recommendations were also highlighted and passed to the Chair of the SOCC on publication of the guideline.

\section{Planned review and updating of the guideline}

In line with BTS policy, this guideline will be reviewed by the SOCC within 5 years of publication.

\section{Declaration of interest}

BTS Declarations of Interest forms have been completed by all members for each year they were part of the GDG. Details of these forms can be obtained from BTS Head Office. Declarations of Interest was a standing item at each GDG meeting.

\section{Stakeholders}

Stakeholders were identified at the start of the process and where appropriate societies and organisations were contacted and asked to nominate a specific person to join the GDG. All stakeholder organisations were notified when the guideline was available for public consultation.

\section{SECTION 3: CLINICAL FEATURES WHICH PREDICT THE PRESENCE OF MESOTHELIOMA}

There is a paucity of evidence exploring clinical features specific for MPM. Many of the studies are retrospective questionnaire-based case series, which possess a major inherent recall bias in the diagnosed group making interpretation difficult.

There is consistency in the following risk factors and clinical features:

- Male preponderance is in keeping with occupational exposure. ${ }^{4}$

- High-risk occupations include production of asbestos sheets, brake and clutch linings, construction/demolition work, dock and ship yard workers, electricians, plumbers and launderers. ${ }^{5}$

- The predicted lifetime risk of mesothelioma for British men born in the 1940s, who did >10 years of work in the following categories, before the age 30 is as below: $5.9 \%$ for carpenters, $2 \%$ for plumbers, electricians and painters and $0.8 \%$ for other construction workers. ${ }^{6}$

- Non-occupational routes of exposure involves: para exposure via a relative or partner, living in the vicinity of an asbestos factory and environmental exposure (low level). ${ }^{4}$ There is a higher risk of developing MPM from exposure to amphiboles (brown and blue asbestos) rather than chrysotile (white asbestos, the most commonly used form). ${ }^{7}$ The mean latency between asbestos exposure and developing the disease is 40 years for pleural and 46 years for peritoneal mesothelioma. 4

- There are rare familial cases linked to mutation of the breast cancer-associated protein 1 (BAP1) gene. ${ }^{8}$

\section{Symptoms}

Chest pain and dyspnoea are the most common presenting symptoms, but the relative frequency of these symptoms is not consistent in different studies. Other symptoms include weight loss, fevers and sweats ${ }^{410}$ (Table 3).

\section{Clinical signs}

Pleural effusion is often present. Other signs are variable (eg, palpable lymph nodes).$^{10}$ Right side predominance of the disease in the order of 1.6:1. This might partially reflect the increased pleural surface area of the right hemithorax. ${ }^{4}$

Usually the first investigation in patients with suspected mesothelioma will be a chest X-ray. The NICE Guideline on Investigation and Referral for Suspected Cancer gives guidance on when a chest X-ray should be offered in suspected MPM (table 4). The CDG noted that smoking and finger clubbing are more appropriate when considering lung cancer, as opposed to mesothelioma. 
Table 3 Symptoms at initial presentation in 90 evaluable cases of malignant pleural mesothelioma ${ }^{10}$

\begin{tabular}{lcc}
\hline Symptom & No. of cases & $\%$ \\
\hline Pain & 62 & 69 \\
\hline \multicolumn{1}{c}{ Non-pleuritic } & 56 & \\
\hline Pleuritic & 6 & \\
\hline Shortness of breath & 53 & 59 \\
\hline Fever, chills or sweats & 30 & 33 \\
\hline Weakness, fatigue or malaise & 30 & 33 \\
\hline Cough & 24 & 27 \\
\hline Weight loss & 22 & 24 \\
\hline Anorexia & 10 & 11 \\
\hline Sensation of heaviness or fullness in chest & 6 & 7 \\
\hline Hoarseness & 3 & 3 \\
\hline Early satiety & 2 & 2 \\
\hline Myalgias & 2 & 2 \\
\hline Others* & 1 each & 1 \\
\hline
\end{tabular}

*Other symptoms included aphonia and dysphagia, abdominal distension, sensation of pressure in right upper quadrant, nausea, bad taste in mouth perceived tachycardia and headache.

\section{Evidence statements}

Occupational exposure to asbestos is recalled in the majority of patients with MPM. High-risk occupations are ship building and construction/demolition work (including boiler repair, and working as a carpenter or electrician). Level $2-$.

Symptoms are not specific to MPM. Common symptoms at presentation include chest pain and breathlessness. Less common symptoms at presentation include weight loss, fatigue, fever and cough. Level 2-.

The most common examination finding at presentation is a pleural effusion (with $<1$ in 10 presenting with lymphadenopathy or clubbing). Level $2-$.

\section{Recommendations}

- Do not rule out a diagnosis of MPM on the basis of symptoms and examination findings alone. Grade D.

- Offer an urgent chest X-ray to patients with symptoms and signs as outlined in NICE NG12. Grade D.
- Refer all patients with a chest X-ray suggestive of MPM urgently (via the 2-week wait suspected cancer pathway in England and Wales). Consider referral for further investigation in patients with persistent symptoms and history of asbestos exposure despite normal chest X-ray. Grade D.

- A thorough occupational history should be taken to cover all occupations throughout life. It is important to elicit para exposure by exploring details of relative and/or partner occupations. Grade D.

\section{SECTION 4: STAGING SYSTEMS}

In 2016, the International Association for the Study of Lung Cancer (IASLC) Staging Committee published proposals for the revisions of the T, N and $\mathrm{M}$ descriptors for the eighth edition of the TNM classification of MPM. ${ }^{11}$ This was an international, multi-institutional cohort study. The study population was patients with newly diagnosed (cytologically or histologically) MPM. Information was collected on the extent of disease, demographic characteristics, comorbidities, treatment and survival. The dataset included data on 1987 patients with pathologically confirmed MPM from 29 centres on four continents. These comprised 509 cases with only clinical staging information, 836 cases with only pathological staging information (ie, surgical staging) and 642 cases with both clinical and pathological information available. Survival was examined for $\mathrm{T}, \mathrm{N}$ and $\mathrm{M}$ categories according to the seventh edition staging system. Categories were then modified where appropriate to improve prognostic performance. Clinical and pathological T1a and T1b were combined into a single T1 classification. Clinical and pN1 and pN2 categories were collapsed into a single $\mathrm{N}$ category comprising ipsilateral, intrathoracic nodal metastases (N1). Nodes previously categorised as N3 were reclassified as N2. M category remained unchanged (see table 5). The proposed TNM groupings are shown in table 6. Figure 1 shows the survival curves for each of the new TNM stage groupings. The prognostic performance comparisons for each stage demonstrated statistically significant $\mathrm{HRs}$ for stage IB versus IA, stage IIIA versus II and stage IV versus IIIB.

The Brigham and Women's Hospital Group proposed an alternative system to the AJCC/UICC staging system. ${ }^{12}$ The alternative system is based on patients undergoing EPP, but this has not been accepted widely nor proposals from it included in AJCC/ UICC staging group.

\begin{tabular}{l}
\hline Table 4 NICE NG 12. Referral criteria for suspected malignant pleural mesothelioma ${ }^{185}$ \\
\hline Offer an urgent chest X-ray (to be performed within 2 weeks) to assess for mesothelioma in people aged 40 and over if \\
They have 2 or more of the following unexplained symptoms, or \\
They have 1 or more of the following unexplained symptoms and have ever smoked, or \\
They have 1 or more of the following unexplained and have been exposed to asbestos: \\
Cough \\
Fatigue \\
Shortness of breath \\
Chest pain \\
Weight loss \\
Appetite loss. [new 2015] \\
Consider an urgent chest X-ray (to be performed within 2 weeks) to assess for mesothelioma in people aged 50 and over with either: \\
Finger clubbing \\
Chest signs compatible with pleural disease. [new 2015] \\
\hline
\end{tabular}

ONICE [2015] Suspected cancer: recognition and referral. Available from www.nice.org.uk/guidance/ng12 All rights reserved. Subject to Notice of rights. NICE guidance is prepared for the National Health Service in England. All NICE guidance is subject to regular review and may be updated or withdrawn. NICE accepts no responsibility for the use of its content in this product/publication. 
Table 5 Eighth edition AJCC/UICC staging for malignant pleural mesothelioma

\begin{tabular}{ll}
\hline Stage & Definition \\
\hline Primary tumour (T) \\
\hline TX & Primary tumour cannot be assessed \\
\hline T0 & No evidence of primary tumour \\
T1 & Tumour limited to the ipsilateral \\
& parietal \pm visceral \pm mediastinal \pm diaphragmatic pleura \\
T2 & Tumour involving each of the ipsilateral pleural surfaces (parietal, \\
& mediastinal, diaphragmatic and visceral pleura) with at least one of \\
& the following features: \\
& Involvement of diaphragmatic muscle \\
& Extension if tumour from visceral pleura into the underlying \\
& pulmonary parenchyma \\
T3 & Describes locally advanced but potentially resectable tumour. Tumour \\
& involving all of the ipsilateral pleural surfaces (parietal, mediastinal, \\
& diaphragmatic and visceral pleura) with at least one of the following \\
& features: \\
& Involvement of endothoracic fascia \\
& Extension into the mediastinal fat \\
& Solitary, completely resectable focus of tumour extending into the \\
& soft tissues of the chest wall \\
& Non-transmural involvement of the pericardium
\end{tabular}

T4 Describes locally advanced technically unresectable tumour. Tumour involving all of the ipsilateral pleural surfaces (parietal, mediastinal, diaphragmatic and visceral pleura) with at least one of the following features:

- Diffuse extension or multifocal masses of tumour in the chest wall, with or without associated rib destruction

- Direct transdiaphragmatic extension of tumour to peritoneum

- Direct extension of tumour to the contralateral pleura

- Direct extension of tumour to mediastinal organs

- Direct extension of tumour into the spine

- Tumour extending through to the internal surface of the pericardium with or without pericardial effusion, or tumour involving the myocardium

Regional lymph nodes (N)

NX Regional lymph nodes cannot be assessed

NO No regional lymph node metastases

N1 Metastases in the ipsilateral bronchopulmonary, hilar or mediastinal (including the internal mammary, peridiaphragmatic, pericardial fat pad or intercostal lymph nodes) lymph nodes

N2 Metastases in the contralateral mediastinal, ipsilateral or contralateral supraclavicular lymph nodes

Distant metastasis (M)

M0 No distant metastasis

M1 Distant metastasis present

Reprinted from Journal of Thoracic Oncology, Vol 11, No 12, Rusch V.W et al, THE IASLC Mesothelioma Staging Project: Proposals for the M Descriptos and for Revision of the TNM Stage Groupings in the Forthcoming (Eighth) Edition of the TNM Classification for Mesothelioma. 2112-2119 (2016), with permission from Elsevier.

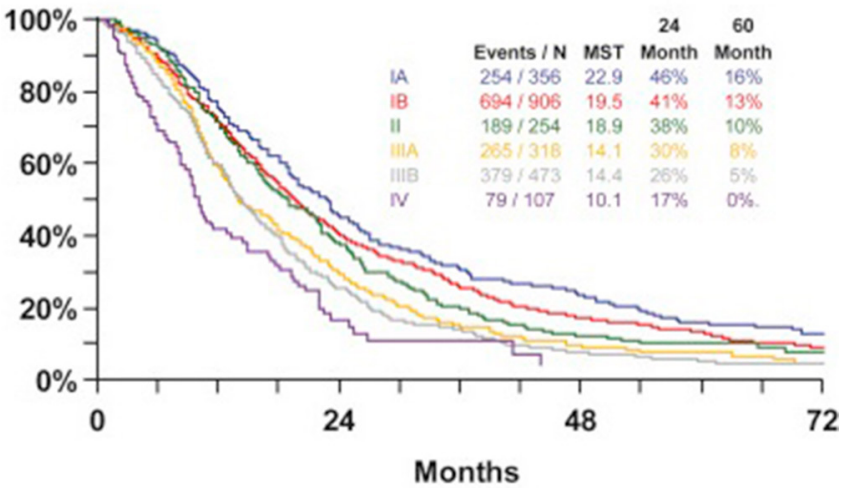

Figure 1 Overall survival according to best stage (proposed eighth edition). Reprinted from Journal of Thoracic Oncology, Vol 11, No 12, Rusch V.W et al, THE IASLC Mesothelioma Staging Project: Proposals for the M Descriptos and for Revision of the TNM Stage Groupings in the Forthcoming (Eighth) Edition of the TNM Classification for Mesothelioma. 2112-2119 (2016), with permission from Elsevier.

The 2016 National Mesothelioma Audit reported that only $42 \%$ of patients with MPM diagnosed in 2014 had stage recorded. ${ }^{1}$

\section{Evidence statements}

The proposed eighth edition of the IASLC TNM staging system predicts survival in surgically and non-surgically treated patients with MPM. Level 3.

\section{Recommendation}

- Record staging of MPM according to the version 8 of the IASLC staging proposals. Grade D.

\section{SECTION 5: IMAGING MODALITIES FOR DIAGNOSING AND STAGING}

The literature search revealed a large volume of evidence assessing the role of several imaging modalities in the diagnosis and staging of MPM. The use of ultrasound, computed tomography (CT), positron emission tomography (PET) and positron emission tomography-computer tomography (PET-CT) and magnetic resonance imaging (MRI) were all included in the literature review.

A large number of the studies were conducted in mainland Europe or North America. Only a small number of studies were from the UK. The imaging characteristics of MPM are likely to be similar across the world and the demographic profile of patients included is similar to that of patients in the UK (male predominance, mean age $>50$ years). Therefore, the evidence was considered applicable to the UK population.

Table 6 TNM stage groupings proposed for the eighth edition of MPM staging system relative to those used in the seventh edition

\begin{tabular}{lllllll}
\hline & N0 & & N1/N2 & N1 & N3 & N2 \\
\hline Stage & Seventh edition & Eighth edition & Seventh edition & Eighth edition & Seventh edition & Eighth edition \\
T1 & I (A, B) & IA & III & II & IV & IIIB \\
T2 & II & IB & III & II & IV & IIIB \\
T3 & II & III & III & IIIA & IV & IIIB \\
T4 & IV & IV & IIIB & IV & IIIB \\
M1 & IV & IV & IV & IV & IV \\
\hline Rep
\end{tabular}

Reprinted from Journal of Thoracic Oncology, Vol 11, No 12, Rusch V.W et al, THE IASLC Mesothelioma Staging Project: Proposals for the M Descriptos and for Revision of the TNM Stage Groupings in the Forthcoming (Eighth) Edition of the TNM Classification for Mesothelioma. 2112-2119 (2016), with permission from Elsevier. 


\section{Evidence on diagnostic imaging}

The majority of diagnostic evidence evaluates the role of imaging in differentiating benign from malignant pleural disease in general, rather than from MPM specifically. Numerous studies have demonstrated the utility of CT, PET-CT and MRI in the assessment of patients with suspected pleural malignancy. ${ }^{13}$ These studies provide clear guidance on standard morphological characteristics of pleural malignancy using CT and $\mathrm{MRI}^{14-17}$ and are summarised in table 7 along with reported sensitivities and specificities. ${ }^{18-22}$

Pleural malignancy is typically unilateral. Bilateral involvement is rare, accounting for as few as $3 \%$ of cases. ${ }^{15}$ In $94 \%$ of cases of pleural malignancy, there is a pleural effusion on the affected side. However, differentiation between MPM and metastatic pleural malignancy can be challenging. The presence of lung parenchymal involvement or mediastinal or hilar lymph node enlargement may help point towards metastatic pleural disease. ${ }^{21}$ While the presence of pleural plaques is an indicator of prior asbestos exposure, it is not a marker of malignancy per se and effusions can be found in this context as a result of benign asbestos-related pleural effusion.

PET-CT can be used to provide useful functional information additional to morphology. Typically, areas of abnormal malignant pleural thickening have elevated maximal standardised uptake values (SUVmax). ${ }^{23} 24$ Thus, using a SUVmax threshold of $>2.0$ has been found to accurately differentiate malignant from benign pleural disease with a sensitivity of $88 \%-100 \%$ and specificity of $88 \%-92 \% .^{25-27}$ In a meta-analysis of 11 PET-CT studies, this technique had a pooled sensitivity of $95 \%$ (95\% CI $92 \%$ to $97 \%$ ) and specificity $82 \%$ (95\% CI $76 \%$ to $88 \%$ ) for differentiation of malignant from benign pleural disease. ${ }^{28}$ Causes of false negatives include: small volume tumours and those with a low proliferative index, for instance, early stage epithelioid mesothelioma. In addition, false positives may result from inflammatory diseases, tuberculous pleurisy, parapneumonic effusions and prior talc pleurodesis. One study, which included patients with prior talc pleurodesis, reported significantly lower specificity in comparison to other studies (specificity $35.3 \%$ ), as a result of the high number of false positives in this group. ${ }^{29}$

Studies using MRI have highlighted its potential in distinguishing benign from malignant pleural disease. Malignant pleural thickening tends to show inhomogenous hyperintensity on proton-density $\mathrm{T} 2$-weighted images and enhancement on T1-weighted images following gadolinium injection, in contradistinction to benign disease that is of low signal on both sequences. When these signal characteristics are combined with morphology and a pleural thickening $>1 \mathrm{~cm}$, the accuracy of MRI is very high for differentiation of benign from malignant disease with sensitivity of $100 \%$ and specificity of $95 \%$ in one study $(95 \%$ confidence intervals not reported). ${ }^{30}$ More recent studies have highlighted potential utility for diffusion-weighted MR imaging (DWI-MRI) in differentiating pleural malignancy from benign pleural disease, with lower Apparent Diffusion Coefficient (ADC) values being demonstrated in pleural malignancy. ${ }^{31}{ }^{32}$ Coolen et $a l$ also performed DWI-MRI in a study of pleural malignancy and reported that inhomogeneous restriction in diffusion of the thickened pleura differentiates malignant from benign pleural disease with a sensitivity of $92.5 \%$ (95\% CI $84 \%$ to $97 \%$ ) and specificity of $79 \%$ (95\% CI $62 \%$ to $89 \%) .{ }^{33}$ Gill et al demonstrated that patients with epithelioid MPM have a significantly higher ADC value than those with non-epithelioid MPM and an ADC threshold of 1.1 could differentiate epithelioid MPM from sarcomatoid MPM with a sensitivity of $60 \%$ and specificity of $94 \%$ (95\% CIs not reported). ${ }^{32}$ These MRI data appear promising but are yet to be validated prospectively and importantly their added value in disease with atypical or equivocal CT signs is unclear.

\section{Evidence on staging}

Seventeen ${ }^{25}{ }^{34-49}$ studies were identified that evaluated the role of various imaging modalities when staging MPM. One systematic review ${ }^{50}$ and one meta-analysis ${ }^{51}$ were also identified in the literature. To a degree all imaging modalities are limited in accuracy of staging compared with the gold standard of postoperative histological staging and mediastinoscopic sampling of lymph nodes. However, assessment of limitations is made difficult by the relative infrequency of surgical resection and the use of comparator imaging techniques as the reference point in many of the studies.

Despite the overall benefits of CT scanning when initially assessing patients with suspected mesothelioma, CT performs poorly when compared against other modalities for staging of MPM. CT is particularly poor at assessing T4 stage where assessment of invasion through soft tissue such as diaphragm and chest wall is required. CT also performs poorly at lymph node staging, particularly when detecting involved $\mathrm{N} 2$ and $\mathrm{N} 3$ nodes. In one study, $37 \%$ of the patients were upstaged following a PET scan. ${ }^{35}$

The role of MRI is limited in staging MPM. ${ }^{34} 363739414248$ However, MRI does perform better than CT, where tumour-soft tissue delineation is required. For example, MRI has a sensitivity and specificity of $87.5 \%$ and $87.5 \%$ for stage II disease, and $91 \%$ and $100 \%$ for stage III disease due to its superiority in detecting invasion into or through chest wall, endothoracic fascia, diaphragmatic muscle and mediastinal fat. ${ }^{36}$ Table 8 provides a brief summary.

\begin{tabular}{|c|c|c|c|}
\hline Morphology & Imaging modality & Sensitivity (\%) & Specificity (\%) \\
\hline Pleural thickening $>1 \mathrm{~cm}$ & CT & $35-47$ & $64-94$ \\
\hline \multirow[t]{2}{*}{ Pleural nodularity } & CT & $37-48$ & $86-97$ \\
\hline & MRI & 48 & 86 \\
\hline \multirow[t]{2}{*}{ Infiltration of the chest wall and/or diaphragm } & CT & $17-29$ & 100 \\
\hline & MRI & 44 & 100 \\
\hline \multirow[t]{2}{*}{ Mediastinal pleural involvement } & CT & $70-74$ & $83-93$ \\
\hline & MRI & 77 & 93 \\
\hline Interlobar fissure nodularity & CT & 10 & 100 \\
\hline
\end{tabular}


Table 8 Showing the sensitivity and specificity of CT, MRI and positron emission tomography (PET)-CT in mesothelioma staging ${ }^{36}$

\begin{tabular}{llllll}
\hline \multirow{2}{*}{$\begin{array}{l}\text { Imaging } \\
\text { modality }\end{array}$} & Stage II & & & Stage III & \\
\cline { 2 - 3 } \cline { 5 - 6 } & Sensitivity (\%) & Specificity (\%) & Sensitivity (\%) & Specificity (\%) \\
\hline CT & 100 & 69.20 & 75 & 100 \\
MRI & 87.50 & 87.50 & 91 & 100 \\
\hline PET-CT & 100 & 100 & 100 & 100 \\
\hline
\end{tabular}

It should also be noted that although Plathow $e a^{36}$ showed an accuracy of $100 \%$ and low interobserver variability when staging patients with MPM with PET-CT, compared with CT and MRI, the results of other smaller studies are mixed.

Pragmatically CT (optimised for pleural enhancement) remains the mainstay of imaging for diagnosis and staging of MPM. A number of centres routinely include the abdomen and pelvis in the initial CT scan whereas others perform completion scanning according to the results of other diagnostic tests.

\section{Evidence statements}

Overall reported diagnostic accuracy of CT in the detection of pleural malignancy is $68 \%-97 \%$, with specificity of $78 \%-89 \%$. Level: 3 .

CT and ultrasound features of malignant pleural disease include pleural thickening $>1 \mathrm{~cm}$, nodular pleural thickening, mediastinal pleural thickening and interlobar fissural nodularity. Level: 3.

Features favouring MPM over metastatic pleural malignancy are the presence of pleural plaques, involvement of the interlobar fissure and the absence of lung parenchymal involvement. Level: 3.

Overall reported diagnostic accuracy of PET-CT in the detection of pleural malignancy-sensitivity $88 \%-95 \%$, specificity 35\%-100\%. Level: $2+$.

False positives at PET-CT are common in TB pleuritis, inflammatory disorders of the pleura and previous talc pleurodesis. Level: 3.

Overall reported diagnostic accuracy of MRI in the detection of pleural malignancy-sensitivity $60 \%-100 \%$, specificity 73\%-95\%. Level: 2-.

CT has limited accuracy for staging MPM using current staging systems. Level: 3 .

MRI is better than CT at detecting invasion through diaphragm and T3 disease (invasion through muscle, bone, mediastinal fat), but has limited sensitivity in nodal staging. Level: 3 .

Integrated PET-CT has the highest accuracy for staging MPM. It has better sensitivity across all three criteria $\mathrm{T}, \mathrm{N}$ and $\mathrm{M}$ compared with CT and MRI. Level: $2+$.

\section{Recommendations}

- Offer CT thorax with contrast (optimised for pleural evaluation) as the initial cross-sectional imaging modality in the evaluation of patients with suspected MPM. Grade D.

- Use of PET-CT for aiding diagnosis of MPM is not recommended in patients who have had prior talc pleurodesis and caution should be employed in populations with a high prevalence of TB. Grade D.

- In patients where differentiating $\mathrm{T}$ stage will change management, consider MRI. Grade D.

- In patients where excluding distant metastases will change management, offer PET-CT. Grade D.

\section{SECTION 6: PATHOLOGICAL DIAGNOSIS}

A diagnosis of MPM can be challenging because the tumour has a wide range of morphological appearances and may mimic many other epithelial or sarcomatoid malignancies. The best method for obtaining pleural tissue is already covered in the current BTS pleural disease guidelines. For this reason, this topic was not covered in the PICOT questions used in our initial mesothelioma literature search. The BTS pleural disease guideline can be downloaded at the following website: https://www.brit-thoracic. org.uk/standards-of-care/guidelines/

In summary, the BTS pleural guideline states:

1. In patients with a symptomatic exudative pleural effusion where a diagnostic pleural aspiration is negative or inconclusive, thoracoscopy (either by local anaesthetic thoracoscopy or VATS) is suggested as the next choice investigation since the procedure is relatively uncomplicated and pleurodesis can be performed at the same time if indicated.

2. If a contrast-enhanced thoracic CT scan of a patient shows a focal area of abnormal pleura (with or without a pleural effusion), an image-guided cutting needle biopsy has a high yield and low complication rates. This technique is particularly useful in patients who are unsuitable for thoracoscopy.

The morphological features of MPM are well described elsewhere in the WHO classification of pleural tumours, ${ }^{52}$ and the guidelines of the International Mesothelioma panel, ${ }^{53}$ and are beyond the scope of this guideline. The importance of histological subtyping of MPM is highlighted in the national mesothelioma audit report, which demonstrates that non-epithelioid histology was associated with significantly shorter overall survival in this cohort. ${ }^{1}$ Table 9 highlights the main subtypes of mesothelioma and the different morphological features that might be present within each group.

The literature search identified 176 papers related to the use of ancillary techniques to improve the diagnosis of malignant mesothelioma. Several were rejected due to study age, the applicability of the diagnostic tests, small numbers of cases, or an inability to extract data, resulting in 70 papers being selected for review. All were retrospective case series. Case numbers varied greatly, from 23 up to 596 cases, and were often very heterogeneous case mixtures. Immunohistochemistry (IHC) was by far the most frequently considered ancillary diagnostic technique. Other approaches used included electron microscopy, chromosomal analysis, microRNA expression, DNA methylation, mRNA expression array, fluid chemistry assay, cytofluorimetry, flow cytometry and fluorescence in situ hybridisation (FISH).

The quality of the evidence reviewed was highly variable. Some of the papers were unique descriptions of unusual diagnostic approaches without comparators. In some studies, the origin of the tumour tissue was not clear and others used autopsy

\begin{tabular}{lll}
\hline Table 9 & Mesothelioma subtypes & \\
\hline Epithelioid & Bisphasic & Sarcomatoid \\
\hline Tubulopapillary & Any combination & Cellular storiform \\
\hline Clear cell & & Desmoplastic \\
\hline Adenomatoid & & Leiomyoid \\
\hline Solid & Chondroid \\
Small cell & Lymphohistiocytoid \\
\hline Pleomorphic & \\
\hline
\end{tabular}


material. Many of the older studies, especially those published prior to 1990 , use clones of primary antibody or other immunohistochemical techniques that are no longer used or available. More recent studies typically used contemporary reagents that are available and applicable in the UK.

\section{Summary of individual immunohistochemistry evidence}

A large number of IHC markers have been reviewed and are summarised in table 10 , with sensitivity and specificity values where available. It should be noted that the sensitivity and specificity of many of these markers are reduced in sarcomatoid MPM, which frequently does not express any of the typical 'mesothelial' markers. In this scenario, expression of keratins may be the only demonstrable feature, which is helpful but non-specific. Additionally, discriminating malignant from benign mesothelial proliferations is not reliable using IHC markers.

\section{Additional techniques}

Wu et $a l^{54}$ examined p16 FISH to discriminate reactive from malignant mesothelium in 60 patients. Hemizygous or homozygous deletion of p16 was not seen in fibrous pleurisy (FP), but was detected in $66.7 \%$ of epithelioid MPM, $87.5 \%$ of biphasic MPM and 100\% of sarcomatoid cases, highlighting potential utility in the differentiation of MPM from FP. Hida et $a l^{55}$ performed BAP1 and p16 FISH in 40 cases of MPM and 20 cases of inflammatory pleuritis. All inflammatory cases and only three mesothelioma cases were negative for both. The presence of BAP1 and or p16 FISH may therefore be helpful in differentiating MPM from benign mesothelial proliferation.

\section{Diagnosis in cytology}

This remains a controversial subject. The reliability of an MPM diagnosis on effusion cytology is highly variable (sensitivity ranging from $16 \%$ to $73 \%{ }^{5657}$ ) and is very much dependent on cytologist experience. Some centres will send clot/cell block sections for the homozygous deletion of the $9 \mathrm{p} 21$ band (p16), which can increase diagnostic certainty.

\section{Evidence statements}

Glut1 IHC and p16 FISH have potential for discriminating benign from malignant mesothelium. Level 3.

Table 10 Summary of immunohistochemistry markers

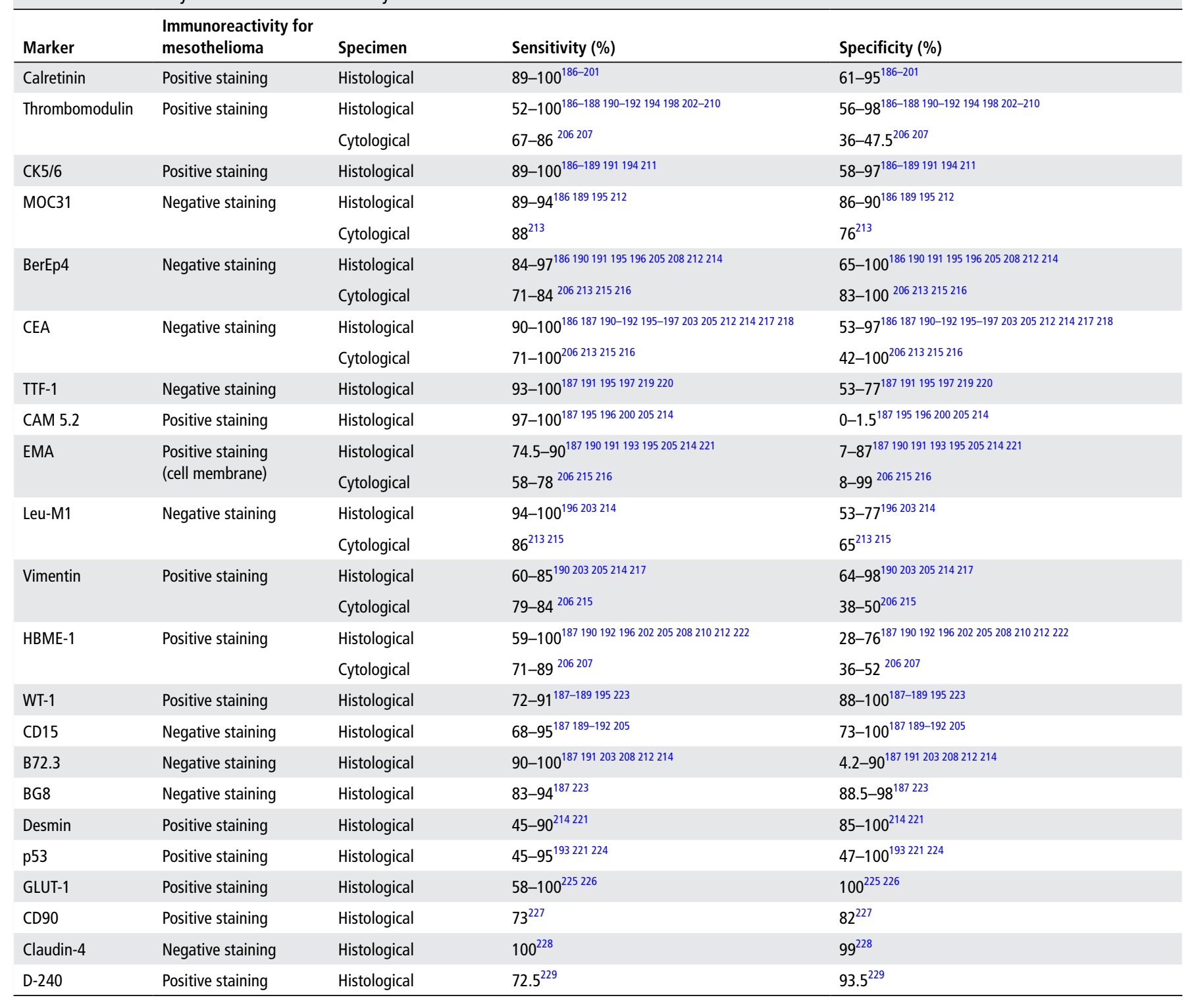


The sensitivity of pleural fluid cytology for the diagnosis of MPM is highly variable and is dependent on the cytologist's experience. Level 3.

Positive IHC markers for MPM include calretinin, thrombomodulin, CK5/6, CAM5.2, EMA, vimentin, GLUT-1, HBME-1, WT-1, p53. Overall sensitivity is $45 \%-100 \%$. Level 3.

Negative IHC markers for MPM include Ber-Ep4, MOC-31, CEA, Leu-1, CD15, TTF-1, B72.3. Overall specificity is 53\%-100\%. Level 3.

A combination of two positive mesothelial markers and two negative adenocarcinoma markers increases diagnostic accuracy. Level 3.

Diagnostic accuracy of IHC markers is reduced in sarcomatoid MPM. Level 3.

Accurate subtyping of IHC markers is reduced in sarcomatoid MPM. Level 3.

\section{Recommendations}

- IHC is recommended for the differential diagnosis of MPM in both biopsy and cytology type specimens. Grade D.

- A combination of at least two positive mesothelial (calretinin, cytokeratin 5/6, Wilms tumour $1, \mathrm{D}-240$ ) and at least two negative adenocarcinoma immunohistochemical markers (TTF1, CEA, Ber-EP4) should be used in the differential diagnosis of MPM. (Markers listed in likely order of value). Grade D.

- Do not rely on cytology alone to make a diagnosis of MPM unless biopsy is not possible or not required to determine treatment due to patient wishes or poor PS. Grade D.

- Pathologists should report the histological subtype of MPM in all cases. Grade D.

\section{Good practice points}

$\checkmark$ Biopsies from patients with suspected MPM should be reviewed by a pathologist experienced in the diagnosis of MPM and a second opinion should be sought if there is uncertainty over the diagnosis.

\section{SECTION 7: USE OF BIOMARKERS}

The literature search revealed a large volume of evidence, exploring different biomarkers that may have a role in MPM. Literature on at least 20 markers tested in serum, plasma, pleural fluid and exhaled breath were reviewed. A number of markers were assessed in exploratory studies with no further validation, and such markers have not been considered further given the lack of validation studies.

Several markers such as Mesothelin, Fibulin-3, Osteopontin and Megakaryocyte Potentiating Factor (MPF) have been extensively studied internationally. Individual studies and controlled meta-analyses specifically looking at these markers were identified and reviewed. Significant heterogeneity was noted between study populations. In particular, there was wide variability in comparator groups and disease prevalence. For example, comparator groups include normal controls, asbestos-exposed well individuals, patients with benign effusions and patients with non-mesothelioma malignant effusions. In some areas, the prevalence of mesothelioma in the sampled population was $>30 \%$, in others $<5 \%$. The cut-off value for markers varied in most studies.

Although most studies included sarcomatoid mesothelioma, this made up only a small proportion of the overall cohort of any single study.

\section{Evidence on diagnostic markers}

The most robust body of evidence at present for diagnosis of MPM is for Soluble Mesothelin Related Peptides (SMRP) and Osteopontin, as summarised below:

- A meta-analysis by Cui et $a l^{58}$ reviewed 28 publications totalling 7550 patients (1562 MPM and 5988 non-MPM), which confirmed serum SMRP to have an overall sensitivity of $60 \%$ and a specificity of $81 \%$, with an area under the curve (AUC) of 0.734 .

- The same review also demonstrated that pleural fluid SMRP has an overall sensitivity of $75 \%$, specificity $76 \%$ and AUC of 0.809 (total number of patients 1506; $460 \mathrm{MPM}$ and 1046 non-MPM).

- Summary sensitivities and specificities for SMRP and Osteopontin-from two meta-analyses by $\mathrm{Hu}$ et al, ${ }^{59}$ reviewing six publications with a total of 906 patients, and Lin et $a l^{60}$ reviewing seven publications with a total of 1096 patients (Table 11).

There were a number of studies on Fibulin-3, representing a smaller body of evidence than that above for SMRP and Osteopontin. These are summarised in table 12.

\section{Markers for disease monitoring and assessment of progression}

Sixteen ${ }^{61-76}$ papers were reviewed in relation to above. Again, SMRP is the most widely studied marker but other biomarkers such as Fibulin-3, Osteopontin, Megakaryocyte potentiating factor (MPF and Hyaluronic acid (HA) were also assessed. Study populations are heterogeneous with regards to their management. Disease progression/stability in these studies has in general been assessed by the use of the modified response evaluation criteria in solid tumours (RECIST).

Overall:

- SMRP shows a positive correlation with tumour bulk. ${ }^{62}$

- In patients who had EPP, there was a significant drop in SMRP levels (on average 54\%). Despite the relationship with tumour bulk, there is no significant correlation with increasing disease stage.

- Mean and median SMRP levels for those with progressive disease showed a significant difference compared with patients with partial/complete response and stable disease. ${ }^{62}$

- A falling SMRP level between baseline and two cycles of chemotherapy was associated with a longer 'time to progression' of disease. Fibulin-3 failed to show a similar relationship. $^{75}$

- Low Fibulin-3 at diagnosis is associated with a prolonged survival. ${ }^{61}$

Table 11 Summary sensitivities and specificities for Soluble Mesothelin Related Peptides (SMRP) and Osteopontin (OPN)

\begin{tabular}{llll}
\hline & & Sensitivity & Specificity \\
\hline SMRP & Serum & $60(95 \% \mathrm{Cl} 56$ to 64$)$ & $81(95 \% \mathrm{Cl} 78$ to 83$)$ \\
& Pleural fluid & $75(95 \% \mathrm{Cl} 69$ to 80$)$ & $76(95 \% \mathrm{Cl} 71$ to 82$)$ \\
& Serum+plasma & $65(95 \% \mathrm{Cl} 60$ to 70$)$ & $81(95 \% 78$ to 85$)$ \\
OPN & Serum+plasma & $57(95 \% \mathrm{Cl} 52$ to 61$)$ & $81(95 \% 79$ to 84$)$ \\
\hline
\end{tabular}


Table 12 Summary sensitivities and specificities for Fibulin-3

\begin{tabular}{|c|c|c|c|c|c|}
\hline & & Sensitivity & Specificity & Area under the curve & Cut-off (ng/mL) \\
\hline \multirow[t]{3}{*}{ Pass et al ${ }^{230}$} & Plasma & 100 & 100 & 1 & $33^{*}$ \\
\hline & Plasma & 95 & 96 & 0.99 & $53 \dagger$ \\
\hline & Pleural fluid & 84 & 92 & 0.93 & 346 \\
\hline \multirow[t]{2}{*}{ Agha et al ${ }^{231}$} & Serum & 88 & 81.8 & 0.776 & 67 \\
\hline & Pleural fluid & 72 & 80 & 0.878 & 150 \\
\hline \multirow[t]{2}{*}{ Elgazzar et al ${ }^{232}$} & Serum & 100 & 97 & 0.98 & 54 \\
\hline & Pleural fluid & 90 & 97 & 0.94 & 520 \\
\hline \multirow[t]{3}{*}{ Creaney et $a l^{233}$} & Plasma & 22 & 95 & $\mathrm{n} / \mathrm{a}$ & 52 \\
\hline & Plasma & 48 & 71 & 0.671 & 29 \\
\hline & Pleural fluid & 59 & 52 & 0.588 & 346 \\
\hline Kirschner et a $a^{61}$ & Plasma & $\begin{array}{l}14 \\
13\end{array}$ & $\begin{array}{l}97 \\
88 \S\end{array}$ & & $29 \ddagger$ \\
\hline
\end{tabular}

${ }^{*}$ Detroit cohort.

†New York cohort.

$\ddagger$ Sydney cohort.

$\S$ Vienna cohort.

\section{Outcome prediction}

Four studies ${ }^{71727475}$ assessed the independent predictive value of biomarkers on overall survival in MPM, accounting for the recognised prognostic indicators of histological subtype, age and PS. These demonstrate:

- The modified Glasgow Prognostic Score (mGPS) (serum, $\mathrm{C}$ reactive protein (CRP) and albumin level at baseline) and blood neutrophil-to-lymphocyte ratio (NLR) are independent predictors of overall survival (HR 2.6 and 2.0, respectively). ${ }^{71}$

- Pleural fluid HA level $(<225 \mathrm{mg} / \mathrm{L})$ is independently associated with overall survival (RR 0.63). ${ }^{72}$

- Resection specimen staining for smoothened SMO transmembrane receptor (HR 1.06) was an independent predictor of overall survival. $^{74}$

A fall in SMRP between baseline and an interval of 6-8 weeks (post two cycles of chemotherapy) is predictive of radiographic stability of disease. A falling SMRP level at completion of chemotherapy is strongly associated with a longer survival. ${ }^{75}$ Baseline SMRP was unable to predict survival. Apart from SMRP in the SWAMP study, ${ }^{75}$ none of the other markers have been prospectively validated.

\section{Biomarkers for screening}

Five studies ${ }^{77-81}$ explored the potential role of biomarkers in screening for MPM. All five studies looked at SMRP but two studies also looked at Osteopontin, CA-125 and cytokeratin fragment $19 .{ }^{58} 75$ Studies were heterogeneous particularly with regards to the cut-off value of SMRP, duration of follow-up and the patient populations (other cancers/control groups). Despite these differences, SMRP tended to be higher in those with asbestos-related disorders such as asbestosis or diffuse pleural thickening, and in renal impairment. One study found SMRP levels are also elevated in other cancers such as lung, ovarian, pancreatic and endometrial cancer, but the populations of patients with these cancers were small.

\section{Evidence statements}

Diagnosis

There is no diagnostic biomarker, which is able to consistently diagnose MPM with a sensitivity and specificity above $90 \%$. Level $2+$.
The diagnostic value of biomarkers in sarcomatoid mesothelioma is lower than that for epithelioid, but small numbers mean that accuracy of sensitivity and specificity are difficult to derive. Level 2-.

Serum SMRP has a relatively high specificity in the diagnosis of MPM across a large number of studies (81\%). Level $2+$.

Serum and pleural fluid Osteopontin has a relatively high specificity in the diagnosis of mesothelioma across a modest number of studies (81\%). Level $2++$.

Fibulin-3 shows variable performance in diagnosis of MPM (sensitivity range 22\%-100\%). Level $2+$.

\section{Disease response}

SMRP level is correlated with tumour bulk and falls post-EPP, but baseline level does not predict pathological stage in mesothelioma. Level $2+$.

In assessing response to therapy, SMRP levels are higher in those with progressive disease compared with those with partial response, complete response or disease stability. Level 3.

During chemotherapy, a falling level of SMRP from baseline to interval, or a falling level at completion of palliative chemotherapy is associated with a longer survival. Level 3.

\section{Outcome prediction}

There is no prospectively validated biomarker which independently predicts overall survival in MPM. Level 2-.

Markers of inflammation, pleural fluid HA and cell staining patterns may predict survival but further studies are required to validate this. Level $2-$.

\section{Recommendations}

- Do not offer biomarkers in isolation as a diagnostic test in MPM. Grade B.

- Consider biomarker testing in patients with suspicious cytology who are not fit enough for more invasive diagnostic tests. Grade B.

- Do not routinely offer biomarker testing to predict treatment response or survival. Grade B.

- Do not offer biomarker testing to screen for MPM. Grade C.

\section{Research recommendation}

Further research is required to identify biomarkers that reliably predict treatment response within clinical practice 


\section{SECTION 8: FACTORS DETERMINING PROGNOSIS AND TIMING OF TREATMENT}

There is a large body of evidence on this topic in the literature. The great majority of it is of poor quality, being retrospective case series. Some of these are taken from patients enrolled into clinical trials, where the consistency and quality of the data collected is higher.

A large number of baseline patient variables have been studied seeking prognostic factors. These include demographic factors (age, sex, race), disease features (histological subtype and grade, site of disease, disease stage using various staging systems), Eastern Cooperative Oncology Group (ECOG) PS or Karnofski performance score (KPS), symptoms (particularly chest pain and weight loss, usually not further defined), markers of inflammation (total white blood count (WBC), platelet count, neutrophil-to-lymphocyte ratio (NLR), platelet-to-lymphocyte ratio (PLR), C-reactive protein level (CRP) and blood test markers of systemic disease such as haemoglobin $(\mathrm{Hb})$ level, $\mathrm{Hb}$ difference from a population ideal value $(160 \mathrm{~g} / \mathrm{L}$ in men, $140 \mathrm{~g} / \mathrm{L}$ in women), serum albumin.

Several prognostic scores have been developed for mesothelioma, combining groups of prognostic variables derived from derivation cohorts of patients with mesothelioma and subsequently validated in different test cohorts. The following scores are described in more detail below; the EORTC prognostic score (EPS), the CALGB score,${ }^{82-87}$ mGPS have been studied retrospectively in a cohort of patients with mesothelioma, ${ }^{71}$ the LENT prognostic score ${ }^{88}$ and a prognostic model using decision tree analysis was published by Brims et $a l^{89}$ in 2016.

\section{Evidence from very large studies}

Three retrospective studies were identified, which included more than 5000 patients from population-level registries. ${ }^{90-92}$ These consistently demonstrate that increasing age, male sex, advanced stage and non-epithelioid histology are prognostic of worse overall survival. Although this evidence is of low quality, being retrospective, the size of the datasets studied and the absence of any contradictory evidence increases the confidence in these findings.

\section{Findings from the National Lung Cancer Audit}

In 2015, Beckett et al published data from 8740 mesothelioma cases included in the National Lung Cancer Audit. ${ }^{3}$ This is the largest prospectively collected case series in the literature. It has the advantage of reflecting the characteristics of unselected incident cases. In this respect, it differs from the populations of clinical trial recruits who have been used to derive, for example, the EORTC and CALGB prognostic scores (see below). Poorer PS and non-epithelioid histology were associated with shorter overall survival in this cohort. Survival by sex is not reported.

\section{The EORTC prognostic score}

This was derived by Curran et al $l^{83}$ in 1998 based on maximum-likelihood parameter estimates of the prognostic factors retained in a multivariate model derived from a population of 204 patients $(89 \%$ male) entered into clinical trials of chemotherapy in Europe. All patients were PS 0-2. More detail on the score can be found at appendix 2 .

\section{CALGB prognostic groups}

Herndon et al studied prognostic factors in a group of 337 patients with MPM not previously treated with chemotherapy who were entered into phase II trials of chemotherapy. ${ }^{87} \mathrm{Cox}$ survival and exponential regression trees were used to determine prognostic importance of pretreatment patient characteristics. Terminal nodes were amalgamated to form six distinct prognostic subgroups.

The derived prognostic groups are complex, and continuous variables are dichotomised differently for different subgroups (eg, $\mathrm{Hb}$ and $\mathrm{WBC}$ ). Edwards et al validated the CALGB groups in a retrospective study conducted in a UK population. ${ }^{86}$

Meniawy et al have validated the CALGB prognostic group method in a recent, large study in Western Australia, in a population of patients where $62 \%$ received chemotherapy. This is considerably higher than the proportion of patients currently receiving chemotherapy for mesothelioma in the UK and therefore the median survival estimates derived from the validation study are likely to be considerably better than those observed in the UK.

\section{The neutrophil-to-lymphocyte ratio}

Five studies have considered the NLR in mesothelioma. The evidence on the prognostic utility of NLR was reviewed by Meniawy et al..$^{85}$ They concluded that the cut-off value chosen for NLR is variable, the independent predictive effect inconsistent and the NLR has not been validated in a prospective study. More information about the studies can be found in appendix 2 .

\section{The modified Glasgow prognostic score}

The mGPS stratifies patients with cancer according to CRP and serum albumin. This was found to be an independent predictor of overall survival in MPM in one study ${ }^{71}$ (HR 2.6, 95\% CI 1.6 to $4.2, \mathrm{P}<0.001$ ) but has not been the subject of prospective validation.

\section{Prognostic model using decision tree analysis}

Brims et al derived a prognostic model using classification and regression tree analysis from an unselected population of 482 patients newly diagnosed with MPM in Western Australia, of whom 274 were collected retrospectively and 208 prospectively. ${ }^{89}$ Unlike the cohorts used to derive the CALGB and EORTC models, which were of participants in chemotherapy trials, this paper included all patients with a confirmed diagnosis of MPM within the inclusion period. The model was validated in a cohort of 177 patients with MPM prospectively collected in Bristol, UK. The validation cohort is likely to be highly representative of typical new patients with MPM presenting in the UK. The model was used to predict death at 18 months. The variable with the greatest influence on survival in the derivation cohort was weight loss, defined as any weight loss considered significant by the medical team. The decision tree for classifying patients into prognostic groups in this study is shown in table 13. The variables having an influence on prognosis within this model are

\begin{tabular}{clc}
\hline Table 13 & Brim decision tree classification & \\
\hline $\begin{array}{l}\text { Prognostic } \\
\text { group }\end{array}$ & $\begin{array}{l}\text { Median survival (IQR), months, } \\
\text { derivation cohort }\end{array}$ & $\begin{array}{l}\text { Median survival (IQR), } \\
\text { months, validation cohort }\end{array}$ \\
\hline 1 & $34.0(22.9-47.0)$ & N/A \\
\hline 2 & $17.7(11.6-25.9)$ & $11.93(8.53-18.56)$ \\
3 & $12.0(6.0-20.6)$ & $9.89(4.84-17.81)$ \\
4 & $7.4(3.3-11.1)$ & $5.68(3.12-10.84)$ \\
\hline
\end{tabular}

N/A, not available. 
histological subtype, weight loss, $\mathrm{PS}, \mathrm{Hb}$ and serum albumin. The C-statistic for the derivation cohort was 0.76 and the sensitivity $94.5 \%$ (95\% CI $91.4 \%$ to $96.7 \%)$ and the specificity $38.2 \%$ (95\% CI $30.6 \%$ to $46.3 \%$ ). The positive predictive value for death at 18 months was $76 \%$ (95\% CI $71.5 \%$ to $80.1 \%)$. The $\mathrm{C}$-statistic for model performance in the validation cohort was 0.68 (95\% CI 0.60 to 0.75 ).

The model can be found in appendix 2 .

\section{The LENT score}

Clive and others derived the LENT score, for predicting survival in patients presenting with malignant pleural effusion (MPE). ${ }^{88}$ The LENT score uses pleural fluid LDH (>1500) IU/L, ECOG PS, NLR and tumour type to calculate a prognostic score (see appendix 3 for a full description of the scoring system). Data from three large international cohorts of patients were used to study the effect of the malignant cell type on survival. A more detailed analysis of individual prognostic factors was then undertaken in two prospectively collected UK cohorts of patients presenting with MPE. One cohort was used to derive a prognostic score and the second to validate it. Fourteen predefined variables, recorded at presentation, were studied to ascertain their influence on survival using a multivariable Cox proportional hazard method. A prognostic score was then developed using the results of the international cohort for cell type and the UK cohort multivariable analysis.

Patients with moderate-risk and high-risk LENT scores had hazard ratios $(95 \% \mathrm{CI})$ for mortality of 1.49 (1.03-2.15) and 5.97 (3.58-9.97) compared with those with low-risk LENT scores. The relation between LENT score and median survival is shown in table 14.

\section{Symptoms}

Chest wall pain and weight loss have been studied as prognostic variables. ${ }^{828587}$ In retrospective case series, chest pain was independently associated with poorer overall survival in all three studies but has not been subjected to prospective validation. The findings with respect to weight loss are inconsistent. Weight loss was independently predictive of survival in two studies ${ }^{85} 87$ but not in the third. ${ }^{82}$

\section{Evidence statements}

Increasing age, male sex, non-epithelioid histology, advanced stage and poorer PS independently predict poorer survival in MPM. Level 2+.

The LENT prognostic score provides an approximate estimate of median survival, at presentation, in patients presenting with a pleural effusion due to MPM. Level $2+$.

The EPS and CALGB prognostic groups reliably separate patients into groups with better and worse overall survival but they have been studied only retrospectively, in patients with better PS and treated with chemotherapy in the majority. Level $2+$.

Markers of inflammation including WBC, platelet count, CRP, serum albumin, PLR and NLR may predict survival, but further studies are required, particularly prospectively, to validate this. Level 3.

\begin{tabular}{lcc}
\hline \multicolumn{2}{l}{ Table 14} & Summary of \\
\hline Risk categories & Total score & Median (IQR) survival \\
\hline Low risk & $0-1$ & 319 days (228-549 days) \\
Moderate risk & $2-4$ & 130 days (47-467 days) \\
High risk & $5-7$ & 44 days (22-77 days) \\
\hline
\end{tabular}

The decision tree model separated unselected UK patients newly diagnosed with MPM into groups with differing median survival using variables that are routinely collected in almost all patients. Level $2+$.

\section{Recommendations}

- Consider calculating a prognostic score in patients with MPM at diagnosis. Grade D.

- Prognostic scores can provide useful survival information for patients and doctors, but should not be used in treatment decision-making. Grade D.

- When calculating a prognostic score use one of the following:

a. The EORTC prognostic score;

b. The CALGB score;

c. The modified Glasgow Prognostic Score (mGPS);

d. The LENT score if a pleural effusion is present;

e. The decision tree analysis.

The decision tree analysis scoring systems is likely to be the most useful in routine clinical practice. Grade D.

\section{SECTION 9: PLEURAL FLUID MANAGEMENT}

There is poor consistency in the literature concerning the outcome of 'pleurodesis success', as it is variably defined according to time point, radiology only, combined radiology and need for further pleural drainage and by patient-reported outcome measures.

There is also substantial lack of consistency in the analysis of time to event data, with many studies reporting proportion of 'success' at a given time point in those patients assessable at the time, that is, patients who have died or are unable to attend follow-up are discounted, leading to increasing rates of pleurodesis success over time in some studies.

Rintoul et al directly compared VATS-PP to talc (poudrage or slurry). Although early pleurodesis success, as assessed by chest X-ray reporting, appeared high in the VATS-PP group, this was not sustained over the study follow-up period $(37 \%$ talc vs $59 \%$ VATS PP at 1 month, $60 \%$ at 3 months in both, $57 \%$ talc vs 77\% VATS PP at 6 months, but 77\% talc vs 70\% VATS $\mathrm{PP}$ at 12 months). ${ }^{93}$ VATS pleurectomy was not associated with survival benefit (primary outcome) nor benefits to lung function. VATS-PP patients had a significantly higher complication rate (31\% vs 14\%) and longer hospital stay (7 vs 3 days). VATS was associated with slight improvement in quality of life, but only from the 6-month follow-up point onwards and not in all quality of life domains.

Davies et al undertook an RCT comparing indwelling pleural catheter (IPC) insertion with talc slurry in patients with symptomatic malignant pleural effusions and found no difference in pleurodesis success or patient measured breathlessness. ${ }^{94}$

Fysh et al undertook a large retrospective case series which demonstrated no difference in surgical versus 'medical' pleurodesis in MPM (28.2\% vs $29.7 \%$ complete success, $39.7 \%$ vs $38.8 \%$ partial success). ${ }^{95}$ In another retrospective series, Bielsa et al demonstrated worse pleurodesis success in mesothelioma $(66 \%)$ and lung (63\%) versus breast $(77 \%)$ and other (74\%). ${ }^{96}$

Two other studies specific to MPM evaluated VATS pleurodesis in non-comparative case series, reporting pleurodesis success rates of $81 \%-98 \%$, but were retrospective, and suffer from selection bias and used different pleurodesis definitions. ${ }^{97} 98$ Non-MPM-specific studies reported pleurodesis success rates of $80 \%-86 \%$ and did not differentiate mesothelioma from other MPE. One of these studies reported PS rather than pleurodesis success. $^{99-101}$ 


\section{Evidence statements}

Pleural effusion due to MPM may have a lower pleurodesis success rate than other malignant effusions. Level 2-.

No single fluid control technique (surgical including pleurectomy and VATS, thoracoscopic talc poudrage, talc slurry or IPC) has been shown to be superior in terms of patient symptoms or pleurodesis success in MPM. Level 1-.

VATS-PP has been shown to be more expensive, associated with greater complications and longer hospital stay than talc slurry pleurodesis. Level $1+$.

VATS-PP is associated with minor improvement in quality of life versus talc slurry in those patients who survive $>6$ months. Level 1-.

Indwelling pleural catheters and talc slurry pleurodesis have similar patient-related outcomes in malignant effusion and MPM. Level $1++$.

\section{Recommendations}

- Offer either talc (via slurry or poudrage) or indwelling pleural catheters for symptomatic pleural effusion in MPM, informed by patient choice. Grade A.

- Talc slurry or thoracoscopic talc poudrage pleurodesis should be offered to patients with MPM in preference to a VATS-PP surgical approach for fluid control in MPM. Grade A.

\section{SECTION 10: THE ROLE OF SURGERY}

Surgical resection has been offered to a highly selected subgroup of patients with MPM since the 1950s, although its role remains controversial. Surgery can be offered with palliative intent, where the aim is debulking of the tumour mass with the aim of controlling pleural fluid, reducing pulmonary restriction or by attempting to achieve a complete macroscopic resection, with the aim of improving length and/or quality of life. The International Association for the Study of Lung Cancer's Staging and Prognostic Factors Committee has proposed definitions for surgery, which have been adopted for this guidance. ${ }^{102}$

1. Partial pleurectomy (PP): partial removal of parietal and/ or visceral pleura for diagnostic or palliative purposes but leaving gross tumour behind. This may be performed by VAT or with thoracotomy.

2. Pleurectomy/Decortication (PD): parietal and visceral pleurectomy to remove all gross tumour without resection of the diaphragm or pericardium.

3. Extended Pleurectomy/Decortication (EPD): parietal and visceral pleurectomy, with the goal of complete macroscopic resection, with resection of the diaphragm and/or pericardium as required.

4. Extrapleural pneumonectomy (EPP): en bloc resection of the parietal pleura, pericardium, diaphragm, lung and visceral pleura.

\section{Evidence review}

95 papers were identified and reviewed, of which 12 were considered in detail. ${ }^{83} 93$ 103-112 There were two RCTs, four systematic reviews, four prospective observational studies and two retrospective studies.

\section{Pleurectomy}

A systematic review has been performed of 34 studies involving 1916 patients who underwent pleurectomy. ${ }^{110}$ These included 12 studies on extended PD, 8 studies on PD and 14 studies on
PP. All the studies were observational with high risk of selection bias. Perioperative mortality ranged from $0 \%$ to $11 \%$ and perioperative morbidity ranged from $13 \%$ to $43 \%$. Median overall survival ranged from 7.1 to 31.7 months and disease-free survival ranged from 6 to 16 months.

The MesoVATS trial randomised 196 patients with suspected or confirmed mesothelioma (of whom 175 had mesothelioma) between talc pleurodesis or VATS-PP. ${ }^{93}$ The primary outcome was survival at 1 year, which was $52 \%$ (95\% CI 41 to 62 ) in the VAT-PP group and 57\% (95\% 46 to 66) in the talc pleurodesis group (HR 1.04 (95\% CI 0.76 to 1.42); $\mathrm{P}=0 \cdot 81$ ). Surgical complications were significantly more common after VAT-PP than after talc pleurodesis, occurring in 24 (31\%) of 78 patients who completed VAT-PP versus $10(14 \%)$ of 73 patients who completed talc pleurodesis $(\mathrm{P}=0.019)$. Median hospital stay was longer at 7 days (IQR 5-11) in patients who received VAT-PP compared with 3 days $(2-5)$ for those who received talc pleurodesis $(\mathrm{P}<0.0001)$.

\section{Extended pleurectomy decortication and Extra-pleural pneumonectomy}

The Mesothelioma and Radical Surgery (MARS) feasibility study assessed EPP versus no EPP for patients with MPM. ${ }^{103}$ Patients with pathologically confirmed mesothelioma deemed fit enough to undergo trimodal therapy were included. All patients underwent induction platinum-based chemotherapy followed by clinical review. After further consent, patients were randomly assigned to EPP followed by postoperative hemithorax irradiation or to no EPP. Of 112 patients registered, 50 were subsequently randomly assigned: 24 to EPP and 26 to no EPP. EPP was completed satisfactorily in 16 of 24 patients assigned to EPP. Two patients in the EPP group died within 30 days and a further patient died without leaving hospital. One patient in the no EPP group died perioperatively after receiving EPP off trial in a non-MARS centre. The HR for overall survival between the EPP and no EPP groups was 1.90 (95\% CI 0.92 to 3.93; exact $\mathrm{P}=0.082$ ), and after adjustment for sex, histological subtype, stage and age the HR was $2.75(95 \%$ CI 1.21 to $6.26 ; \mathrm{P}=0 \cdot 016)$. Median survival was 14.4 months (5.3-18.7) for the EPP group and 19.5 months (13.4 to time not yet reached) for the no EPP group. Of the 49 randomly assigned patients who consented to quality of life assessment (EPP $n=23$; no EPP $n=26$ ), 12 patients in the EPP group and 19 in the no EPP group completed the quality of life questionnaires. Although median quality of life scores were lower in the EPP group than the no EPP group, no significant differences between groups were reported in the quality of life analyses.

There has been much discussion around the validity of the MARS trial results. In particular, criticism that the study was not powered to detect a survival advantage attributable to EPP and that the operative mortality was higher than that of other contemporary series. The MARS trial authors have subsequently responded that the EPP mortality in MARS (2 of 19; $10.5 \%$; $95 \%$ confidence limits $1.3 \%$ to $33.1 \%$ ) lies within the range reported in a systematic review of 34 studies, including 2320 patients, where 30 -day mortality ranged from $0 \%$ to $11.8 \% .{ }^{113}$ Furthermore, the authors note that the median survival of patients in the EPP arm of MARS of 14.4 months from randomisation is in keeping with major series in the literature which report median survival times of 10 to 14 months.

Cao $e t a l^{108}$ performed a systemic review of 34 studies with 2462 patients who underwent EPP for MPM. All the studies were observational and subject to high risk of selection bias. The median overall survival varied from 9.4 to 27.5 months, and 1-year, 2-year and 5-year survival rates ranged from 36\% 
to $83 \%, 5 \%$ to $59 \%$ and $0 \%$ to $24 \%$, respectively. Overall perioperative mortality rates ranged from $0 \%$ to $11.8 \%$, and the perioperative morbidity rates ranged from $22 \%$ to $82 \%$. Quality of life assessments from three studies reported improvements in nearly all domains at 3 months postoperatively. Patients who underwent trimodality therapy involving EPP and adjuvant chemoradiotherapy had a median overall survival of 13-23.9 months.

Two meta-analyses have been performed comparing outcomes following either PD or EPP. All the studies included in the analyses were observational with high risk of selection bias. The meta-analysis by Taioli et al ${ }^{114}$ included 1512 patients treated with PD and 1391 treated with EPP. There was a significantly higher proportion of short-term deaths in the EPP group versus the PD group (per cent mortality meta-estimate; $4.5 \%$ vs $1.7 \%$; $\mathrm{P}<0.05)$. There was no statistically significant difference in 2 -year mortality between the two groups, but there was significant heterogeneity. The meta-analysis by Cao et al $(2014)^{111}$ included 632 patients who underwent EPP and 513 patients who underwent EPD. ${ }^{111}$ All-cause perioperative mortality was found to be significantly lower after EPD compared with EPP (2.9\% vs $6.8 \%$; RR 0.53 ; $95 \%$ CI 0.31 to $0.91 ; \mathrm{P}=0.02$; $\mathrm{I}^{2}=0 \%$ ). Perioperative morbidity was also found to be significantly lower after EPD compared with EPP (27.9\% vs 62.0\%; RR 0.44; 95\% CI 0.30 to $\left.0.63 ; \mathrm{P}<0.0001 ; \mathrm{I}^{2}=44 \%\right)$. There were insufficient data for this meta-analysis to compare the overall survival outcomes between the two treatment arms.

The effects of PD on lung function and quality of life have been assessed in a number of small cohort studies. None of these studies compared changes in outcomes with patients who were not selected to undergo surgery and so the results must be interpreted with caution. Mollberg et al found that quality of life scores did not deteriorate in 28 patients with good PS (0-1) who underwent PD. ${ }^{104}$ Bölükbas et al found that the mean FVC improved from $55 \%$ of predicted to $69 \%$ of predicted $(\mathrm{P}<0.01)$ in 16 patients who underwent radical pleurectomy. ${ }^{105}$ Ploenes et al retrospectively reviewed the outcomes of 25 patients who underwent EPP and 23 who had PD. ${ }^{107}$ Pulmonary function was not significantly reduced in the PD group postoperatively. In the EPP group, the median vital capacity fell from $78 \%$ of predicted to $48 \%$ predicted $(\mathrm{P}<0.001)$. Burkholder et al assessed quality of life in 36 patients undergoing PD. ${ }^{106}$ Global quality of life scores were unchanged in the 17 patients with PS of 0 and improved in the 19 patients with PS of 1 or 2 .

A feasibility multicentre randomised trial comparing EPD to no surgery (MARS-2 trial) is currently recruiting in the UK. ${ }^{112}$ Results from this surgical trial are awaited with interest.

\section{Evidence statements}

VAT-PP has no effect on overall survival and results in increased complications and longer hospital stay than talc pleurodesis. Level $1+$.

EPP does not improve survival when added to treatment with chemoradiotherapy. Level $1+$.

EPD may result in lower perioperative mortality than EPP. Level 1-.

Quality of life and lung function may not deteriorate in patients selected to undergo pleurectomy decortication. Level $2-$.

\section{Recommendations}

- Do not offer VATS-PP over talc pleurodesis in MPM. Grade A.

- Do not offer EPP in MPM. Grade B.

- Do not offer EPD outside of a clinical trial. Grade D.
Research recommendation

The role of VATS-PP and EPD in good prognosis patients should be examined further in clinical trials, which should include robust measurement of quality of life.

\section{SECTION 11: SYSTEMIC ANTICANCER TREATMENT Evidence}

The literature search revealed a large volume of evidence assessing the role of systemic treatment. Over two hundred articles were obtained from a search. Of these, 69 were not relevant to the question. Papers were excluded if they involved trimodality therapy or radiotherapy as major features in the trial design. This included papers looking at the role of neoadjuvant or adjuvant chemotherapy in the setting of surgery. Papers were excluded if they involved intrapleural chemotherapy and photodynamic therapy during as part of surgical therapy.

\section{Evidence on first-line systemic therapy}

Almost all the first-line studies identified were non-randomised phase II trials. Four large phase III randomised trials comparing novel systemic therapy to 'standard' therapy were identified. Two of the large randomised trials used a control arm of single-agent cisplatin and one used a control arm of active symptom control (ASC). Table 15 summarises phase III trial data.

The first large randomised trial (known as EMPHASIS) to be published in patients with MPM compared 3 weekly intravenous chemotherapy with the antifolate drug pemetrexed at a dose of $500 \mathrm{mg} / \mathrm{m}^{2}$ and cisplatin at a dose of $75 \mathrm{mg} / \mathrm{m}^{2}$ with a control arm of cisplatin at a dose of $75 \mathrm{mg} / \mathrm{m}^{2}{ }^{215}$ The primary outcome was survival. Secondary outcomes were time to progressive disease, time to treatment failure, tumour response rate and duration of response. 226 patients were randomised to pemetrexed/cisplatin, and 222 to cisplatin alone. The median survival time for pemetrexed/cisplatin-treated patients was longer than for patients receiving cisplatin alone: 12.1 vs 9.3 months, representing a statistically significant difference $(\mathrm{P}=0.020)$. The median time to progressive disease was significantly longer for patients who received pemetrexed and cisplatin as compared with patients who received cisplatin alone (5.7 vs 3.9 months; $\mathrm{P}=0.001$ ). The median time to treatment failure was also significantly longer in the pemetrexed/cisplatin arm than in the control arm. The response rates were $41 \%$ for pemetrexed/cisplatin patients vs $17 \%$ in the control group.

While this trial was recruiting, the investigators became aware of excessive bone marrow toxicity likely due to folate depletion probably caused by pemetrexed. They decided to give all patients, both in the trial arm and the control arm, vitamin B12 (by intramuscular injection) and folic acid (by tablet) supplementation.

Table 15 Randomised phase III trials in first-line treatment of malignant pleural mesothelioma

\begin{tabular}{llllc}
\hline Trial & $\begin{array}{l}\text { Year of } \\
\text { publication }\end{array}$ & $\begin{array}{l}\text { Treatment } \\
\text { arms }\end{array}$ & OS (months) & P value \\
\hline Vogelzang $^{115}$ & 2003 & P/C vs C & 12.1 vs 9.3 & 0.020 \\
van Meerbeeck $^{116}$ & 2005 & R/C vs C & 11.4 vs 8.8 & 0.048 \\
Muers $^{117}$ & 2008 & $\begin{array}{l}\text { ASC+ctx vs } \\
\text { ASC }\end{array}$ & 8.5 vs 7.6 & 0.290 \\
Zalcman $^{119}$ & 2015 & P/C/B vs P/C & 18.8 vs 16.1 & 0.017 \\
\hline
\end{tabular}

ASC, active symptom control; B, bevcizumab; C, cisplatin; ctx, chemotherapy: OS, overall survival; $P$, pemetrexed; $R$, ralitrexed. 
Bone marrow toxicity was reduced and vitamin supplementation is now standard for all patients treated with pemetrexed. The incidence of nausea, vomiting, fatigue, diarrhoea, dehydration and stomatitis were significantly higher in the pemetrexed/ cisplatin arm.

In 2005, a broadly similar RCT was published by the European Organisation for the Research and Treatment of Cancer (EORTC). ${ }^{116}$ The experimental arm was the antifolate drug raltitrexed combined with cisplatin (arm B), with a control group of single-agent cisplatin ( $\operatorname{arm~A}$ ). Raltitrexed is comparable to pemetrexed in that its main mechanism of action is by inhibiting thymidylate synthase, thereby preventing the formation of precursor pyrimidine nucleotides. End points were overall survival, response rates and quality of life. Patients had to have good PS (WHO 0-2) and adequate haematological, renal and hepatic function. Two hundred and fifty patients were randomised: $80 \%$ were male and the median age was 58 years. The main grade 3 or 4 toxicities observed were neutropaenia and emesis, reported twice as often in the combination arm. Among 213 patients with measurable disease, the response rate was $13.6 \%(\operatorname{arm~A})$ vs $23.6 \%$ ( $\operatorname{arm~B} ; \mathrm{P}=0.056)$. Median overall and 1-year survival in arms $\mathrm{A}$ and $\mathrm{B}$ were 8.8 (95\% CI 7.8 to 10.8 ) vs 11.4 months (95\% CI 10.1 to 15$)$, respectively, and $40 \%$ vs $46 \%$, respectively ( $\mathrm{P}=0.048)$.

A large cooperative group based in the UK led by Muers et al organised a large three-arm randomised clinical trial known as MS01. ${ }^{117}$ Patients were randomised into three groups. Group 1: ASC. The essential elements of ASC were defined as regular follow-up in a specialist clinic; structured physical, psychological and social assessments at every clinic visit; rapid involvement of additional specialists and parallel nursing support. Patients could receive, as required, steroids, analgesic drugs, appetite stimulants, bronchodilators or palliative radiotherapy. Group 2: ASC plus mitomycin, cisplatin and vinblastine chemotherapy (MVP), or group 3: ASC plus vinorelbine chemotherapy. A total of 840 patients (280 in each group) were needed to detect an improvement of 3 months survival; however, due to slow accrual the trial design changed to a two-group comparison by combining the two chemotherapy groups. The two-group design needed a total of 420 patients (140 ASC, 280 ASC plus chemotherapy) to reliably detect an improvement from 9 months median survival with ASC alone to 12 months with ASC plus chemotherapy. Four hundred and nine patients with malignant pleural mesothelioma, from 76 centres in the UK and 2 in Australia, were randomly assigned to ASC alone $(n=136)$; to ASC plus MVP (four cycles of mitomycin $6 \mathrm{mg} / \mathrm{m}^{2}$, vinblastine $6 \mathrm{mg} / \mathrm{m}^{2}$ and cisplatin $50 \mathrm{mg} / \mathrm{m}^{2}$ every 3 weeks $(n=137)$ ) or to ASC plus vinorelbine (one injection of vinorelbine $30 \mathrm{mg} / \mathrm{m}^{2}$ every week for 12 weeks $\left.(n=136)\right)$. The results showed that, compared with ASC alone, there was no significant survival benefit for ASC plus chemotherapy (HR $0.89,95 \%$ CI 0.72 to $1.10 ; \mathrm{P}=0.29)$. Median survival was 7.6 months in the ASC-alone group and 8.5 months in the ASC plus chemotherapy group. There were no between-group differences in four predefined quality of life subscales (physical functioning, pain, dyspnoea and global health status) at any of the assessments in the first 6 months. The trial attracted some criticism for the decision to combine the two different chemotherapy arms, thus reducing the power to detect a significant difference for the separate regimens. ${ }^{118}$

A more recent trial reported by Zalcman et al presented data on the addition of bevacizumab to pemetrexed and cisplatin chemotherapy for patients with newly diagnosed MPM. ${ }^{119}$ The trial, called MAPS (Mesothelioma Avastin Cisplatin Pemetrexed
Study) was a randomised, controlled, open-label, phase III trial. Patients aged 18-75 years with unresectable MPM who had not received previous chemotherapy, had an Eastern Cooperative Oncology Group performance status of $0-2$, had no substantial cardiovascular comorbidity, were not amenable to curative surgery, had at least one evaluable (pleural effusion) or measurable (pleural tumour solid thickening) lesion with CT and a life expectancy of $>12$ weeks from 73 hospitals in France. Patients were stratified by histology (epithelioid vs sarcomatoid or mixed histology subtypes), PS score (0-1 vs 2), study centre or smoking status (never smokers vs smokers)) to receive intravenously $500 \mathrm{mg} / \mathrm{m}^{2}$ pemetrexed plus $75 \mathrm{mg} / \mathrm{m}^{2}$ cisplatin with (PCB) or without (PC) $15 \mathrm{mg} / \mathrm{kg}$ bevacizumab in 21-day cycles for up to six cycles, until progression or toxic effects. The primary outcome was overall survival (OS) in the intention-to-treat population. 448 patients were randomised to treatment (223 to PCB and 225 to $\mathrm{PC}$ ). Overall survival was significantly longer with PCB (median 18.8 months (95\% CI 15.9 to 22.6$)$ ) than with PC (16.1 months (95\% CI 14.0 to 17.9); HR 0.77 (95\% CI 0.62 to 0.95$)$; $\mathrm{P}=0.0167)$. Overall, 158 (71\%) of 222 patients given PCB and 139 (62\%) of 224 patients given PC had grade 3-4 adverse events. More grade 3 events, higher rates of hypertension and more thrombotic events were noted with PCB compared with PC. Bevacizumab treatment is not currently licensed for use in the UK and is not available in the NHS.

An International Expanded Access Programme (EAP) led by Santoro followed more than 3000 patients with mesothelioma treated with single-agent pemetrexed or pemetrexed in combination with cisplatin or carboplatin. ${ }^{120}$ Patients with histologically confirmed MPM, not amenable to curative surgery, received pemetrexed $500 \mathrm{mg} / \mathrm{m}^{2}$ in combination with either cisplatin $75 \mathrm{mg} / \mathrm{m}^{2}$ or carboplatin AUC 5, once every 21 days with standard premedication. A total of 1704 chemonaïve patients received pemetrexed plus cisplatin $(n=843)$ or pemetrexed plus carboplatin $(n=861)$ and were evaluated for safety. The efficacy evaluable population consisted of 745 patients in the pemetrexed plus cisplatin group and 752 patients in the pemetrexed plus carboplatin group for whom physician-reported tumour response was available. The pemetrexed plus cisplatin group demonstrated a response rate of $26.3 \%$ compared with $21.7 \%$ for the pemetrexed plus carboplatin group, with similar 1-year survival rates $(63.1 \%$ vs $64.0 \%)$ and median time to progressive disease (7 vs 6.9 months). Based on these data, pemetrexed plus carboplatin is generally considered an acceptable alternative two-drug first-line option, especially for patients deemed unfit for cisplatin, although the data on which this practice is based are not from an RCT.

\section{Second-line systemic treatments in MPM}

Buikhuisen et al undertook a systematic review of 10 studies reporting on 1251 patients treated with second-line chemotherapy in MPM. ${ }^{121}$ The majority of studies were phase II with only two phase III randomised trials. The authors concluded that only a limited number of randomised studies with combination therapy had been conducted. The authors suggested the following as second-line treatment options for patients with MPM: 'single agent vinorelbine or pemetrexed are acceptable second-line agents for patients relapsing after a first-line platinum combination regardless of whether or not pemetrexed was used in the first-line setting'. They also stated that the 'low reported activity of the drugs in second line warrants referral of fit patients to participate in clinical trials'. 
Jassem et al compared the efficacy and safety of pemetrexed and best supportive care in patients with MPM after first-line chemotherapy (excluding pemetrexed). ${ }^{122}$ Of the 243 patients included, $18.7 \%$ of the 143 patients receiving pemetrexed showed a partial response but the median overall survival was not significantly different between the two groups.

The VANTAGE-014 study compared vorinostat, an oral histone deacetylase inhibitor, with placebo in 661 patients with MPM who had previously received one or two systemic regimens. ${ }^{123}$ Median overall survival for vorinostat was 30.7 weeks (95\% CI 26.7 to 36.1 ) vs 27.1 weeks (95\% CI 23.1 to 31.9 ) for placebo (HR $0.98,95 \%$ CI 0.83 to $1.17, \mathrm{P}=0.86$ ).

Anti-PD1 immune checkpoint therapy has potential for the treatment of mesothelioma. Approximately $40 \%$ of tumours express PDL1, which is associated with non-epithelioid histology and worse outcome for high expressing tumours. ${ }^{124}$ Keynote 28 is the first phase Ib trial to report on the activity of pembrolizumab in patients with pleural mesothelioma and enrolled 25 patients harbouring PDL1-positive tumours. ${ }^{125}$ This study showed a $20 \%$ response rate with durability lasting on average 12 months. Stable disease was 52\% giving a disease control rate of $72 \%$. Median overall survival was 18 months. In summary, emerging data suggest anti-PD1 or PDL1 immunotherapy, exhibits efficacy in mesothelioma; however, randomised trials will be needed to confirm the incremental benefit and value. In this regard, the CRUK CONFIRM phase III trial is currently randomising patients $2: 1$ to nivolumab versus placebo (NCT03063450).

\section{Evidence statements}

For patients with MPM with good PS, first-line chemotherapy with cisplatin and pemetrexed leads to longer survival than cisplatin alone. Evidence level $1++$.

For patients with MPM with good PS, first-line therapy with cisplatin and pemetrexed and bevacizumab leads to longer survival than cisplatin and pemetrexed alone. Evidence level 1 ++ .

For patients with MPM with good PS, first-line chemotherapy with cisplatin and raltitrexed leads to longer survival than cisplatin alone. Evidence level $1++$.

The combination of mitomycin, cisplatin and vinblastine or single agent vinorelbine did not demonstrate survival benefit over active symptom control. Evidence level $1+$.

Carboplatin in combination with pemetrexed is a safe and effective alternative to cisplatin in combination with pemetrexed. Evidence level 3.

Second-line pemetrexed does not improve survival in patients previously treated with first-line chemotherapy regimens that did not include pemetrexed. Evidence level 1+.

Second-line vorinostat does not improve survival in patients previously treated with one or two cycles of chemotherapy. Evidence level 1+.

\section{Recommendations}

- Offer patients with MPM with good PS (WHO 0-1) firstline therapy with cisplatin and pemetrexed. Where licensed (not presently in the UK), bevacizumab should be added to this regime. Raltitrexed is an alternative to permetrexed. Grade A.

- Do not offer pemetrexed or vorinostat as second-line treatment for patients with MPM. Grade A.

\section{Good practice points}

$\checkmark$ Where cisplatin is contraindicated, or has adverse risk, offer carboplatin in combination with pemetrexed.

$\checkmark \quad$ First-line clinical trials are an appropriate option for patients with good PS and are recommended above any other option for second-line treatment, provided the patient is of adequate PS.

\section{Research recommendations}

The role of immunotherapy in MPM should be further assessed in large phase III RCTs.

Further RCTs of second-line therapy on MPM are required.

\section{SECTION 12: RADIOTHERAPY}

\section{Prophylactic radiotherapy to procedure tracts}

Subcutaneous tumour nodules, seeded up the tract of previous needle or tube insertions, surgical or other invasive procedures, are sometimes observed in patients with MPM. Prophylactic radiotherapy to these sites may have a role in preventing the development of tumour tract nodules.

\section{Evidence review}

Four RCTs comparing prophylactic radiotherapy to procedure tracts to no radiotherapy, and a systematic review (written before the 2016 RCT was published) are evaluated. ${ }^{126-130}$ The study by Boutin et al was conducted in the era before chemotherapy was routinely offered to patients with MPM fit enough to receive it. ${ }^{126}$ All patients had both an Abrams biopsy and a thoracoscopy before randomisation. The incidence of metastatic nodules in the control group was high (40\%) and has not been replicated in any other observational studies. The studies by Bydder et al and O'Rourke et al excluded patients who had received prior chemotherapy. ${ }^{127} 128$ Information regarding subsequent chemotherapy treatment was not available. The incidence of chest wall nodules in the control groups were lower and the differences in the incidence of nodules between treatment groups not significantly different. It has been questioned whether these studies were adequately powered. ${ }^{130}$

The SMART trial was a randomised, multicentre, phase III trial evaluating whether prophylactic radiotherapy reduces the incidence of procedure tract metastases after surgical and large bore pleural procedures. ${ }^{129}$ Eligible patients were recruited from 22 UK hospitals and randomised (1:1) to immediate radiotherapy (21 Gy in three fractions over three working days), or deferred radiotherapy (same dose given if a procedure tract metastasis (PTM) developed). Two hundred three patients were randomised (102 to immediate radiotherapy, 101 to deferred radiotherapy). No statistically significant difference was identified in the PTM rates of the immediate and deferred radiotherapy groups $(9 / 102$ (8.8\%) vs $16 / 101$ (15.8\%), respectively; OR 0.51 , 95\% CI 0.19 to $1.32 ; \mathrm{P}=0.14)$. There was no difference identified in quality of life, chest pain, analgesia requirements or survival of the two groups.

A Phase III Randomised Trial of Prophylactic Irradiation of Tracts in Patients with Malignant Pleural Mesothelioma Following Invasive Chest Wall Intervention (the PIT trial) was due to complete recruitment in June 2016 and results are expected in $2017 .{ }^{131}$ Table 16 provides a summary of trials comparing prophylactic and procedure tracts with no radiotherapy. 


\begin{tabular}{|c|c|c|c|c|c|c|}
\hline Study & Patients & Treatments & $\begin{array}{l}\text { Nodules in } \\
\text { treatment group }\end{array}$ & $\begin{array}{l}\text { Nodules in control } \\
\text { group }\end{array}$ & Significance & Notes \\
\hline Boutin $1995^{126}$ & 40 & $\begin{array}{l}21 \mathrm{~Gy} \text { in } 3 \\
12.5-15 \mathrm{MeV}\end{array}$ & $0 / 20$ & $8 / 20$ & $P<0.001$ & Prechemotherapy era \\
\hline Bydder $2004^{127}$ & 43 (58 sites) & $10 \mathrm{~Gy}$ in $19 \mathrm{MeV}$ & $2 / 28$ & $3 / 30$ & N.S. & Chemotherapy patients excluded \\
\hline$O^{\prime}$ Rouke $2007^{128}$ & 61 & $\begin{array}{l}21 \mathrm{~Gy} \text { in } 3 \\
250 \mathrm{kV} \text { photons or 9-12 MeV }\end{array}$ & $4 / 31$ & $3 / 30$ & N.S. & Chemotherapy patients excluded \\
\hline Clive $2016^{129}$ & 203 & $21 \mathrm{~Gy}$ in three fractions & 9/102 & $16 / 101$ & N.S. & Chemotherapy included \\
\hline
\end{tabular}

N.S., not significant

\section{Evidence statements}

Three out of four RCTs did not show a reduction in procedure tract metastases with prophylactic radiotherapy to chest wall procedure tracts. Level $1+$.

Prophylactic radiotherapy to chest wall procedure tract has not been shown to improve quality of life, chest pain, analgesia requirements or survival. Level $1+$.

\section{Recommendation}

- Do not offer prophylactic radiotherapy to chest wall procedure tracts routinely. Grade A.

\section{Radiotherapy as part of multimodality treatment}

The role of radiotherapy as part of the multimodality treatment of MPM is controversial. Radiotherapy can be delivered either as the sole local treatment modality after chemotherapy or as an adjuvant/neoadjuvant treatment in the context of a surgical approach. However, as MPM typically involves large areas of the pleura, the delivery of radical doses of radiotherapy are limited by the surrounding organs at risk such as normal lung, liver, heart and spinal cord.

A number of important remarks should be made with regards to the interpretation of the available literature. First, the majority of studies identified evaluated multimodality treatment and very few investigated specifically the role of preoperative/ postoperative radiotherapy or radiotherapy alone. Second, the majority of the studies identified evaluated RT in the context of EPP, which is now very rarely performed in the UK. Lastly, none of the studies reviewed included surgical or radiotherapy quality assurance. Specifically, the majority of the studies reviewed had no built-in radiation dose constraints for organs at risk.

\section{Evidence review}

Twenty-one studies were identified which included radiotherapy as part of the multimodality treatment. ${ }^{103} 132-151$ One evaluated preoperative radiotherapy (in the context of EPP), ${ }^{132} 2$ hemithoracic radiotherapy alone ${ }^{133134}$ and 17 postoperative radiotherapy (4 in the context of pleurectomy decortication and 13 in the context of EPP).

Four studies were retrospective cohort series and 16 were prospective studies, of which only 4 are multicentre and 2 are RCTs.

Studies evaluating postoperative radiotherapy either after EPP or PD have shown that radiotherapy in the context of multimodality treatment is feasible, but some severe toxicities, particularly pneumonitis have been reported. ${ }^{103} 135-150$ The rate of grade 5 radiation pneumonitis ranges from $0 \%$ to $46 \%$ in the studies that have reported radiotherapy-related toxicity and a lung dose-volume effect was identified in patients who developed grade 3 + radiation pneumonitis. ${ }^{135} 140$ 142-144

Only one RCT specifically evaluated the role of postoperative radiotherapy and showed no benefit for this treatment. ${ }^{150}$ The Swiss Group for Clinical Cancer Research (SAKK) trial is a two-part multicentre randomised phase II study, analysed on intention to treat. It included patients with pathologically confirmed MPM, resectable TNM stages T1-3 N0-2, M0, WHO PS $0-1$ and age $<70$ years. In part 1 of the study, patients were given three cycles of neoadjuvant chemotherapy followed by EPP; the primary end point was complete macroscopic resection (R0-1). In part 2, patients with complete macroscopic resection were randomly assigned to receive adjuvant radiotherapy or not (three-dimensional conformal radiotherapy or intensity-modulated radiotherapy was permitted with dose ranging from 55.9 to $57.6 \mathrm{~Gy}$, using a boost technique). The primary end point was locoregional relapse-free survival. One hundred fifty-one patients were evaluable after neoadjuvant chemotherapy, of whom $75 \%$ had EPP and 64\% complete macroscopic resection. Fifty-four patients were enrolled in part 2. Median locoregional relapse-free survival from surgery was 7.6 months (95\% CI 4.5 to 10.7$)$ in the no radiotherapy group and 9.4 months $(95 \% \mathrm{CI}$ 6.5 to 11.9 ) in the radiotherapy group. Median overall survival calculated from registration for patients in part 2 was 20.8 months (95\% CI 14.4 to 27.8 ) in the no radiotherapy group and 19.3 months (95\% CI 11.5 to 21.8 ) in the radiotherapy group. One patient died of grade 5 radiation pneumonitis. However, it should be noted the trial was terminated earlier than planned due to slow accrual (at $73 \%$ of the accrual).

\section{Evidence statements}

Postoperative radiotherapy after chemotherapy and EPP has not been shown to improve survival. Level $1+$.

Postoperative radiotherapy after chemotherapy and pleurectomy decortication has not been shown to improve survival. Level 2-.

Preoperative radiotherapy has not been shown to improve survival. Level 2-.

Radical radiotherapy used in isolation has not been shown to improve survival. Level 2-.

\section{Recommendation}

- Do not offer preoperative or postoperative radiotherapy in MPM. Grade A.

\section{Research recommendation}

Prospective clinical trials of preoperative radiotherapy, postoperative radiotherapy after pleurectomy decortication and definitive radiotherapy after chemotherapy in MPM are required. 


\section{Radiotherapy for symptom palliation}

Symptoms in MPM include pain, breathlessness and cough. Palliative radiotherapy has been used in an attempt to control these symptoms, as well as for other indications.

\section{Evidence review}

There are six studies, of which two explore whole hemithorax irradiation ${ }^{133} 152$ and four of localised treatment to areas of disease and/or symptoms. ${ }^{153-156}$ There are two systematic reviews addressing the role of radiotherapy for symptom palliation. ${ }^{157158}$

Of the hemithorax studies, a retrospective case series described no change in chest pain or PS in 47 patients treated with 40 Gy in 20 fractions. ${ }^{133}$ The other was a prospective phase II study without controls, including 19 patients treated with $30 \mathrm{~Gy}$ in 10 fractions. ${ }^{152}$ It reported an improvement in pain control in $68 \%$ at 1 month, but this was not maintained (1). Toxicity was not reported in this study.

The localised treatment studies showed variable response rates (in terms of pain improvement). The dose and duration of response were also variable in these uncontrolled reports. The results are summarised in table 17.

A randomised phase II study opened to recruitment in the UK in August 2016 aiming to establish optimal dose/fractionation for symptom control in MPM (SYSTEMS2 SRCTN12698107).

\section{Evidence statements}

Hemithorax radiotherapy has not been shown to have a consistent impact on chest pain or PS in MPM. Level 3.

Localised radiotherapy can improve pain control in MPM, although the effect is variable and is short lived. Level 3.

Radiation dose fractionation utilised in studies of localised radiotherapy for pain control in MPM are variable. The optimal dose is not known. Level 3.

\section{Recommendations}

- Do not offer hemithorax radiotherapy for MPM. Grade D.

- Consider palliative radiotherapy for localised pain in MPM where the pain distribution matches areas of underlying disease. Grade D.

Research recommendation

Further prospective randomised clinical trials are required to determine the role of radiotherapy for symptom control in MPM and the optimal dose fractionation.

\section{SECTION 13: SYMPTOM CONTROL}

Review of the literature revealed that there are no randomised controlled studies of symptom control in patients with MPM only.

There is one published case series of 53 patients with pain from MPM managed with cervical cordotomy. ${ }^{159}$ This was a retrospective case note review and although the majority of patients appeared to have a reduction in pain following the procedure, this study is subject to considerable selection and recall bias.

\section{Evidence statement}

There are no studies of symptom control that specifically relate to MPM.

\section{Good practice point}

$\checkmark$ Symptoms in MPM should be managed as per current guidelines for cancer in general (table 18) and early involvement of palliative care specialists is recommended.

Table 17 Summary of studies exploring localised hemithorax irradiation

\begin{tabular}{|c|c|c|c|c|c|}
\hline Study & Type of study & Patients & Dose; number of fractions (\#) & Pain improvement $\%$ & Duration of response \\
\hline Macleod $^{153}$ & $\begin{array}{l}\text { Prospective phase II } \\
\text { No control }\end{array}$ & 40 & 20 Gy; 5\# & 47 & 5 weeks \\
\hline Davis ${ }^{154}$ & Retrospective & 111 & $\begin{array}{l}<20 \mathrm{~Gy}^{*} \\
>40 \mathrm{~Gy}^{*}\end{array}$ & $\begin{array}{l}60 \\
57\end{array}$ & No data \\
\hline Graaf-Strukowska ${ }^{155}$ & Retrospective & 189 & $\begin{array}{l}<4 \mathrm{~Gy} ; 1 \# \\
36 \mathrm{~Gy} ; 9 \#\end{array}$ & $\begin{array}{l}40 \\
50\end{array}$ & $\begin{array}{l}98 \text { days } \\
69 \text { days }\end{array}$ \\
\hline Jenkins ${ }^{156}$ & Retrospective & 54 & 36 Gy; 12\# & 57 & 2 weeks \\
\hline
\end{tabular}

* Fractionation not specified.

\section{Table 18 Summary of current cancer-related symptom management guidelines in relation to common symptoms seen in MPM}

\begin{tabular}{lll}
\hline Symptom & Management & Reference literature \\
\hline Breathlessness & Pleural fluid control & See 'Pleural fluid management' section \\
& Sustained release morphine & 234235 \\
& Breathing control and use of fans & $236-239$ \\
\hline Pain & Opioids & 240241 \\
& Amitriptyline, duloxetine, gabapentin or pregabalin for neuropathic pain & 242243 \\
& Radiotherapy for refractory localised pain & See 'Radiotherapy' section \\
Fatigue & Aerobic exercise & 244 \\
Anorexia & Megestrol acetate & 245 \\
\hline
\end{tabular}




\section{SECTION 14: CARE AND MANAGEMENT Care in multidisciplinary teams}

Multidisciplinary team (MDT) meetings are an established feature in cancer services. Widespread adoption and development, despite very little supporting evidence, has been seen across all tumour types over the last two decades. There is a suggestion that MDT working improves recruitment to clinical trials $^{160}$ and that patients find MDT working reassuring and improves their experience of care. ${ }^{161} 162$

To support the development of MDTs, the National Cancer Action Team published Guidelines on Characteristics of an Effective MDT (NCAT 2010), although given the mesothelioma incidence the option of virtual MDT working should be considered. ${ }^{163}$ NHS England has outlined their commissioning expectations for mesothelioma and recommended the establishment of specialist mesothelioma MDTs which should manage a minimum of 25 patients per year (NHS England 2013).

Bibby $e a^{164}$ recently published a retrospective evaluation of their specialist regional mesothelioma MDT based on the southwest of England. ${ }^{164}$ Of the 210 cases that were reviewed by the specialist MDT, $10 \%$ had their diagnoses overturned and 20\% were enrolled into a clinical trial.

\section{Evidence statement}

Specialist MPM multidisciplinary meetings may improve diagnostic accuracy and recruitment to clinical trials. Evidence Level 3.

\section{Recommendation}

- Consider referring MPM cases to a regional mesothelioma MDT. Grade D.

\section{Good practice points}

$\checkmark$ All mesothelioma cases should be discussed in a timely fashion by a MDT that reviews a sufficient number of cases to maintain expertise and competence in the diagnosis and treatment of MPM.

$\checkmark$ The MDT membership should fulfil the requirements set by national cancer peer review (to include a named clinical nurse specialist for MPM).

$\checkmark \quad$ The MDT should maintain an up-to-date portfolio of mesothelioma trials and offer recruitment to all eligible patients.

\section{Information needs of patients}

Patients undergoing investigation and treatment for mesothelioma may have unmet psychosocial and information needs. A clear understanding is essential for patients and their carers to make informed choices about the options for management. They may need professional support when interpreting information. The NICE guideline on the management of lung cancer (CG121) made detailed recommendations on the information and support needs of patients, some of which will be applicable to MPM. ${ }^{165}$ The National Lung Cancer Forum for Nurses has emphasised the key role of the lung clinical nurse specialist in providing information and support to patients and has produced specific guidance for managing patients with mesothelioma https://www. nlcfn.org.uk/. There are 14 mesothelioma-specific clinical nurse specialists in the UK.

\section{Evidence review}

The search revealed 13 abstracts potentially relevant to this question. Eight studies were of sufficient quality and relevance to be included in the review, of which 4 included fewer than 30 patients; therefore, the volume of evidence is limited. The studies can be grouped in those assessing emotional support, compensation and intervention.

\section{Emotional support}

Granieri et $a l^{166}$ collected quality of life data from 27 patients with MPM, 55 relatives and 40 healthy controls in Italy. ${ }^{166}$ Patients with MPM had a greater belief that goals could not be reached or problems solved, while often claiming that they were more indecisive than the healthy controls. First-degree relatives reported lower opinions of others, a greater belief that goals cannot be reached or problems solved, support for the notion that they are indecisive and were more likely to suffer from fear that significantly inhibited normal activities than were healthy controls. Arber and Spencer ${ }^{167}$ interviewed 10 patients with MPM from two hospitals in the South of England ${ }^{167}$ All participants reported high levels of uncertainty and feelings of a lack of control leading to psychosocial distress since receiving their diagnosis. All the participants found it difficult to cope with their diagnosis because of all the negative information and 'bad news' around MPM, and this led to feelings of despair. Clayson et $\mathrm{al}^{168}$ interviewed 15 patients in the North of England. ${ }^{168}$ Four main themes emerged: coping with symptoms, the burden of medical interventions, finding out about mesothelioma and psychosocial issues. Dyspnoea was the the most common symptom and the unpredictability and often speed of onset caused great distress. All patients acknowledged asbestos as the cause of their disease.

A systematic literature review ${ }^{169}$ comparing psychological care needs of patients with mesothelioma and those with advanced lung cancer found similarities between the two populations but recommend developing separate assessment and care pathways so that distinct differences (hopelessness, legal and financial matters, attribution of blame) can be addressed.

\section{Intervention}

Moore et $a l^{170}$ evaluated a hospital-based mesothelioma support group in London. Six responses were received from 21 attendees. ${ }^{170}$ All of those that responded found the group useful in terms of sharing experiences and gaining information.

\section{Compensation}

Chamming's et al $^{171}$ performed a linked database study in 2407 patients in France and determined that 30\% of patients with MPM did not claim occupational disease compensation. ${ }^{171}$ Claims were lower in older patients, women and white collar workers. A similar study by Cree $e t$ al $l^{172}$ of 568 patients with MPM in Canada demonstrated that only $42 \%$ filed a claim. ${ }^{172}$ A retrospective case note review ${ }^{173}$ performed in North America identified 16 patients with mesothelioma treated at three Department of Veteran Affairs hospitals of whom only one had documented advice on compensation. ${ }^{173}$

Every serious illness creates extra costs for patients and their families and mesothelioma is no exception. Mesothelioma is usually caused by exposure to asbestos. The industrial nature of mesothelioma means patients often have complex benefit and compensation claims.

There are two main ways to get additional financial support when someone is diagnosed with mesothelioma in the UK:

A. State benefits;

B. Pursuing a civil compensation claim. 
For all civil claims, there is a 3-year time limit from the first date the patient became aware that there is evidence of a compensable asbestos-related disease.

\section{State benefits}

The Department for Work and Pensions recognises the seriousness of mesothelioma and does not normally require a medical examination. Patients under the age of 65 years are eligible for the personal independent payment (PIP) and attendance allowance, if the patient is $>65$ years. PIP provides financial assistance for patients who need help with daily living including personal care and mobility. For patients who have been given a terminal diagnosis they can claim under the Special Rules meaning they will be given priority in the claim being dealt with. Under the Special Rules, patients can receive the allowance at the highest rate. An award of these benefits does not affect an individual's right to apply for other means tested benefits.

\section{Industrial injuries disablement benefit}

This is a non-means tested allowance which patients can claim if on the balance of probability they were exposed to asbestos at work. It is not necessary for a person to have worked directly with asbestos to get this benefit. This benefit is paid via direct debit weekly, fortnightly or every 13 weeks. An award of industrial injuries disablement benefit will be treated as income and may affect other means tested benefits.

\section{Pneumoconiosis (Workers Compensation) Act 1979}

This government scheme is designed to compensate those patients exposed to asbestos through work. A lump sum payment under the Pneumoconiosis (Workers Compensation) Act 1979 (PWCA) can be applied for if on the balance of probability the asbestos exposure occurred during their time at work.

\section{Diffuse mesothelioma scheme 2008}

If patients are unable to make a claim under the PWCA, and are not entitled to compensation from an MOD (Ministry of Defence) scheme, a one-off lump sum can be applied for. This is suitable where exposure is from a secondary source, exposure was in the environment, for those who were self-employed or where exposure cannot be specified but occurred in the UK. The lump sum is assessed by the patient's age.

A claim can be made for the lump sum by the deceased's widow or widower, a child aged under 16 years, a partner who was living with the patient with mesothelioma at the time of death or any other relatives who were financially dependent on the patient at the time of death. The amount paid in posthumous claims is lower than in lifetime benefits.

\section{War disablement pension}

If a patient was exposed during their service in the armed forces prior to 1987 , they are not able to make a claim from their employer because the crown has immunity. A claim can however be made from the Service Personnel and Veterans Agency. All veterans can make a choice between receiving a traditional war pension or a lump sum regardless of age at diagnosis.

\section{Civil claim against a previous employer}

If on the balance of probability exposure to asbestos was from an employer or a previous employer, a civil claim can be pursued via a specialist solicitor who deals with asbestos claims. Claims are often made through the insurers of the company by establishing an employer's negligence or breach of statutory duty to protect the worker from the effects of asbestos dust and fibres. If a company or an insurer cannot be found, an application to The 2014 Diffuse Mesothelioma Payment scheme can be made.

As part of a civil claim, the solicitor may be able to recover costs such as pain and suffering or hospice care. All cases are fast tracked with an aim that patients can receive compensation in their lifetime. The vast majority of cases are settled without going to court. Careful discussion from a specialist solicitor with the patient and family is required because some claims are worth more if the patient has died.

\section{Evidence statement}

Patients with MPM and their relatives have reduced quality of life compared with healthy controls. Level $2+$.

A diagnosis of MPM causes high levels of psychosocial distress. Level qualitative.

Documentation of compensation advice and subsequent claims are variable. Level 3.

\section{Recommendations}

- Offer accurate and understandable information to patients and carers about compensation for MPM. Grade D.

- Offer patients with MPM and their carers the opportunity to discuss concerns regarding their disease. Grade D.

\section{Follow-up strategies}

The literature search did not reveal any evidence pertaining to who and how patients with MPM should be followed up. The search identified 12 papers that were thought to be relevant to the imaging component of this question. Following review of the 12 abstracts, 9 papers ${ }^{174-182}$ were fully critiqued to answer the question regarding the best form of imaging when following up patients with MPM.

None of the papers reviewed was from the UK, but a large number were from within the European region. The rest from Australia, the USA and Turkey. Given the patient populations are generally similar, this evidence is broadly applicable to the UK population. Most of the studies are from the prepemetrexed cisplatin chemotherapy era but for the purpose of answering the specific question here about follow-up, the results are generally acceptable.

The papers reviewed were consistent in their findings that a bidimensional method of assessing tumour volume is inadequate in MPM. ${ }^{182}$ A number of the studies compared Response Evaluation Criteria In Solid Tumors (RECIST) with mRECIST CT criteria. Modified RECIST, despite having its limitations, remains the best method of assessing tumour response when followed up over a period of time. ${ }^{174} 183$

One study demonstrated using mRECIST criteria in MRI can be better at soft tissue/tumour delineation and pleural effusion identification, when compared with mRECIST criteria in CT. ${ }^{184}$

Three studies explored the role of volumetric assessment (using Cavalieri principle) of the tumour on CT. ${ }^{146} 177178$ No significant intraclass or interobserver variability noted, but this method is a time consuming and onerous way of measuring tumour in MPM therefore limiting its clinical utility.

\section{Evidence statements}

CT scanning using modified response evaluation criteria in solid tumours (RECIST) for interpretation gives the best assessment of tumour response to chemotherapy. Level 3. 


\section{Recommendation}

- In patients with MPM where accurate determination of radiological progression is required, consider CT with mRECIST measurement. Grade D.

\section{Good practice point}

\section{$\checkmark$ A personalised care approach should be considered for each} patient.

Patients should be offered 3-4 monthly follow-up appointments with an oncologist, respiratory physician or specialist nurse according to their current treatment plan. If patients wish to be seen less frequently, offer regular telephone follow-up with specialist nurse with an option to attend clinic in the event of clinical deterioration.

\section{Author affiliations}

${ }^{1}$ Department of Respiratory Medicine, University Hospitals Birmingham, NHS Foundation Trust, Birmingham, UK

${ }^{2}$ Respiratory, Queen Alexandra Hospital, Portsmouth, UK

${ }^{3}$ Respiratory Medicine, University Hospitals of Leicester, Leicester, UK

${ }^{4}$ Academic Respiratory Unit, North Bristol NHS Trust, Bristol, UK

${ }^{5}$ North Bristol NHS Trust, Bristol, UK

${ }^{6}$ Sheffield Teaching Hospitals, Sheffield, UK

'Division of Cancer Services, University of Manchester, Manchester, UK

${ }^{8}$ University of Leicester \& University Hospitals of Leicester, Leicester, UK

${ }^{9}$ The Park Medical Practice, Shepton Mallet, Somerset, UK

${ }^{10}$ University of Aberdeen, Pathology, Aberdeen, UK

${ }^{11}$ Department of Thoracic Surgery, Glenfield Hospital, Leicester, UK

${ }^{12}$ North Tyneside General Hospital, North Shields, UK

${ }^{13}$ Oxford NIHR Biomedical Research, University of Oxford, Oxford, UK

${ }^{14}$ Papworth Hospital, Thoracic Oncology, Cambridge, UK

${ }^{15}$ Cancer, St Bartholomew's Hospital, London, UK

${ }^{16}$ Respiratory Medicine, Queen Elizabeth University Hospital, Glasgow, UK

${ }^{17}$ Academic Respiratory Unit, Bristol Medical School, University of Bristol, Bristol, UK

Contributors IW and NM were the lead authors with overall responsibility for the full guideline. All named authors contributed to drafting the full guideline on which this summary is based and approved the guideline for submission.

Competing interests $L D$ has received funding from Irwin Mitchell and Lilly Oncology. DF has received funding from Roche, MSD, Lilly, BI, BMS, Bayer and Astex. SH has received from GSK, Chiesi, Astra Zeneca, Mediconf, Pfizer, Sandoz, Napp. KK has received funding from AZ, BI, BMS, Lilly, Merck, MSD, Novartis, Pfizer, Roche. NM has received funding from $B D$. NR has received funding from Rocket Medical.

Provenance and peer review Not commissioned; internally peer reviewed.

(c) Article author(s) (or their employer(s) unless otherwise stated in the text of the article) 2018. All rights reserved. No commercial use is permitted unless otherwise expressly granted.

\section{REFERENCES}

1 National Lung Cancer Audit: Pleural Mesothelioma Report: Royal College of Physicians, 2016

2 British Thoracic Society Standards of Care Committee. BTS statement on malignant mesothelioma in the UK, 2007. Thorax 2007;62:ii1-ii19.

3 Beckett P, Edwards J, Fennell D, et al. Demographics, management and survival of patients with malignant pleural mesothelioma in the National Lung Cancer Audit in England and Wales. Lung Cancer 2015;88:344-8.

4 Yates DH, Corrin B, Stidolph PN, et al. Malignant mesothelioma in south east England: clinicopathological experience of 272 cases. Thorax 1997;52:507-12

5 Aguilar-Madrid G, Robles-Pérez E, Juárez-Pérez CA, et al. Case-control study of pleural mesothelioma in workers with social security in Mexico. Am I Ind Med 2010;53:241-51

6 Peto J. Health and Safety Executive. RR696: Occupational, domestic and environmental mesothelioma risks in Britain. 2009.

7 Bourdès V, Boffetta P, Pisani P. Environmental exposure to asbestos and risk of pleural mesothelioma: review and meta-analysis. Eur J Epidemiol 2000;16:411-7.

8 Cigognetti M, Lonardi S, Fisogni S, et al. BAP1 (BRCA1-associated protein 1) is a highly specific marker for differentiating mesothelioma from reactive mesothelial proliferations. Mod Pathol 2015:28:1043-57.

9 Tanrikulu AC, Abakay A, Kaplan MA, et al. A clinical, radiographic and laboratory evaluation of prognostic factors in 363 patients with malignant pleural mesothelioma. Respiration 2010;80:480-7.
10 Adams VI, Unni KK, Muhm JR, et al. Diffuse malignant mesothelioma of pleura. Diagnosis and survival in 92 cases. Cancer 1986;58:1540-51.

11 Rusch VW, Chansky K, Kindler HL, et al. The IASLC Mesothelioma Staging Project: Proposals for the M Descriptors and for Revision of the TNM Stage Groupings in the Forthcoming (Eighth) Edition of the TNM Classification for Mesothelioma.J Thorac Oncol 2016;11:2112-9.

12 Sugarbaker DJ, Strauss GM, Lynch TJ, et al. Node status has prognostic significance in the multimodality therapy of diffuse, malignant mesothelioma. J Clin Oncol 1993;11:1172-8

13 Hallifax RJ, Haris M, Corcoran JP, et al. Role of CT in assessing pleural malignancy prior to thoracoscopy. Thorax 2015;70:192-3.

14 Salonen O, Kivisaari L, Standertskjöld-Nordenstam CG, et al. Computed tomography of pleural lesions with special reference to the mediastinal pleura. Acta Radiol Diagn 1986;27:527-31.

15 Seely JM, Nguyen ET, Churg AM, et al. Malignant pleural mesothelioma: computed tomography and correlation with histology. Eur J Radiol 2009;70:485-91.

16 Okten F, Köksal D, Onal M, et al. Computed tomography findings in 66 patients with malignant pleural mesothelioma due to environmental exposure to asbestos. Clin Imaging 2006;30:177-80.

17 Knuuttila A, Kivisaari L, Kivisaari A, et al. Evaluation of pleural disease using MR and CT. With special reference to malignant pleural mesothelioma. Acta Radiol 2001;42:502-7.

18 Metintas M, Ucgun I, Elbek 0, et al. Computed tomography features in malignant pleural mesothelioma and other commonly seen pleural diseases. Eur I Radiol 2002:41:1-9.

19 Hierholzer J, Luo L, Bittner RC, et al. MRI and CT in the differential diagnosis of pleural disease. Chest 2000;118:604-9.

20 Leung AN, Müller NL, Miller RR. CT in differential diagnosis of diffuse pleural disease. AJR Am J Roentgenol 1990;154:487-92.

21 Yilmaz $\mathrm{U}$, Polat $\mathrm{G}$, Sahin $\mathrm{N}$, et al. CT in differential diagnosis of benign and malignant pleural disease. Monaldi Arch Chest Dis 2005;63:17-22.

22 Qureshi NR, Rahman NM, Gleeson FV. Thoracic ultrasound in the diagnosis of malignant pleural effusion. Thorax 2009;64:139-43.

23 Elboga U, YIImaz M, Uyar M, et al. The role of FDG PET-CT in differential diagnosis of pleural pathologies. Rev Esp Med Nucl Imagen Mol 2012;31:187-91.

24 Porcel JM, Hernández P, Martínez-Alonso M, et al. Accuracy of fluorodeoxyglucosePET imaging for differentiating benign from malignant pleural effusions: a metaanalysis. Chest 2015:147:502-12.

25 Bénard F, Sterman D, Smith RJ, et al. Metabolic imaging of malignant pleural mesothelioma with fluorodeoxyglucose positron emission tomography. Chest 1998:114:713-22.

26 Abe Y, Tamura K, Sakata I, et al. Clinical implications of 18F-fluorodeoxyglucose positron emission tomography/computed tomography at delayed phase for diagnosis and prognosis of malignant pleural mesothelioma. Oncol Rep 2012:27:333-8

27 Yildirim H, Metintas M, Entok E, et al. Clinical value of fluorodeoxyglucose-positron emission tomography/computed tomography in differentiation of malignant mesothelioma from asbestos-related benign pleural disease: an observational pilot study. J Thorac Oncol 2009:4:1480-4.

28 Treglia G, Sadeghi R, Annunziata S, et al. Diagnostic accuracy of 18F-FDG-PET and $\mathrm{PET} / \mathrm{CT}$ in the differential diagnosis between malignant and benign pleural lesions: a systematic review and meta-analysis. Acad Radiol 2014;21:11-20.

29 Coolen J, De Keyzer F, Nafteux P, et al. Malignant pleural disease: diagnosis by using diffusion-weighted and dynamic contrast-enhanced MR imaging--initial experience. Radiology 2012:263:884-92.

30 Boraschi P, Neri S, Braccini G, et al. Magnetic resonance appearance of asbestosrelated benign and malignant pleural diseases. Scand I Work Environ Health 1999;25:18-23.

31 Revelli M, Chiesa F, Del Prato A, et al. Role of respiratory-triggered diffusion-weighted MRI in the assessment of pleural disease. Br J Radiol 2016. doi:10.1259/bjr.20160289. [Epub ahead of print 29 Jun 2016].

32 Gill RR, Umeoka S, Mamata H, et al. Diffusion-weighted MRI of malignant pleural mesothelioma: preliminary assessment of apparent diffusion coefficient in histologic subtypes. AJR Am J Roentgenol 2010;195:W125-W130.

33 Coolen J, De Keyzer F, Nafteux P, et al. Malignant pleural mesothelioma: visual assessment by using pleural pointillism at diffusion-weighted MR imaging. Radiology 2015:274:576-84.

34 Giesel FL, Bischoff H, von Tengg-Kobligk H, et al. Dynamic contrast-enhanced MRI of malignant pleural mesothelioma: a feasibility study of noninvasive assessment, therapeutic follow-up, and possible predictor of improved outcome. Chest 2006:129:1570-6.

35 Wilcox BE, Subramaniam RM, Peller PJ, et al. Utility of integrated computed tomography-positron emission tomography for selection of operable malignant pleural mesothelioma. Clin Lung Cancer 2009;10:244-8.

36 Plathow C, Staab A, Schmaehl A, et al. Computed tomography, positron emission tomography, positron emission tomography/computed tomography, and magnetic 
resonance imaging for staging of limited pleural mesothelioma: initial results. Invest Radiol 2008;43:737-44.

37 Stewart D, Waller D, Edwards J, et al. Is there a role for pre-operative contrastenhanced magnetic resonance imaging for radical surgery in malignant pleural mesothelioma? Eur J Cardiothorac Surg 2003;24:1019-24.

38 Schouwink JH, Kool LS, Rutgers EJ, et al. The value of chest computer tomography and cervical mediastinoscopy in the preoperative assessment of patients with malignant pleural mesothelioma. Ann Thorac Surg 2003;75:1715-8.

39 Heelan RT, Rusch VW, Begg CB, et al. Staging of malignant pleural mesothelioma: comparison of CT and MR imaging. AJR Am J Roentgenol 1999:172:1039-47.

40 Niccoli-Asabella A, Notaristefano A, Rubini D, et al. 18F-FDG PET/CT in suspected recurrences of epithelial malignant pleural mesothelioma in asbestos-fibersexposed patients (comparison to standard diagnostic follow-up). Clin Imaging 2013:37:1098-103.

41 Knuuttila A, Halme M, Kivisaari L, et al. The clinical importance of magnetic resonance imaging versus computed tomography in malignant pleural mesothelioma. Lung Cancer 1998;22:215-25.

42 Patz EF, Shaffer K, Piwnica-Worms DR, et al. Malignant pleural mesothelioma: value of $\mathrm{CT}$ and MR imaging in predicting resectability. AJR Am J Roentgenol 1992;159:961-6.

43 Erasmus JJ, Truong MT, Smythe WR, et al. Integrated computed tomography-positron emission tomography in patients with potentially resectable malignant pleural mesothelioma: Staging implications. J Thorac Cardiovasc Surg 2005;129:1364-70.

44 Fiore D, Baggio V, Sotti G, et al. Imaging before and after multimodal treatment for malignant pleural mesothelioma. Radiol Med 2006;111:355-64.

45 Flores RM, Akhurst T, Gonen M, et al. Positron emission tomography defines metastatic disease but not locoregional disease in patients with malignant pleural mesothelioma. J Thorac Cardiovasc Surg 2003;126:11-15.

46 Frauenfelder T, Kestenholz P, Hunziker R, et al. Use of Computed Tomography and Positron Emission Tomography/Computed Tomography for Staging of Local Extent in Patients With Malignant Pleural Mesothelioma. J Comput Assist Tomogr 2015;39:160-5.

47 Genestreti G, Moretti A, Piciucchi S, et al. Prognostic value of 18F-FDG standard uptake value by integrated PET/CT in the staging of malignant pleural mesothelioma. Technol Cancer Res Treat 2012;11:163-7.

48 Martini K, Meier A, Opitz I, et al. Diagnostic accuracy of sequential co-registered $\mathrm{PET}+\mathrm{MR}$ in comparison to PET/CT in local thoracic staging of malignant pleural mesothelioma. Lung Cancer 2016;94:40-5.

49 Pinelli V, Roca E, Lucchini S, et al. Positron Emission Tomography/Computed Tomography for the Pleural Staging of Malignant Pleural Mesothelioma: How Accurate Is It? Respiration 2015;89:558-64.

50 Zahid I, Sharif S, Routledge T, et al. What is the best way to diagnose and stage malignant pleural mesothelioma? Interact Cardiovasc Thorac Surg 2011;12:254-9.

51 Sharif S, Zahid I, Routledge T, et al. Does positron emission tomography offer prognostic information in malignant pleural mesothelioma? Interact Cardiovasc Thorac Surg 2011;12:806-11.

52 Galateau-Salle F, Churg A, Roggli V, et al. The 2015 World Health Organization Classification of Tumors of the Pleura: Advances since the 2004 Classification. $J$ Thorac Oncol 2016;11:142-54.

53 Husain AN, Colby T, Ordonez N, et al. Guidelines for pathologic diagnosis of malignant mesothelioma: 2012 update of the consensus statement from the International Mesothelioma Interest Group. Arch Pathol Lab Med 2013;137:647-67.

54 Wu D, Hiroshima K, Matsumoto S, et al. Diagnostic usefulness of p16/CDKN2A FISH in distinguishing between sarcomatoid mesothelioma and fibrous pleuritis. Am J Clin Pathol 2013;139:39-46.

55 Hida T, Matsumoto S, Hamasaki M, et al. Deletion status of $p 16$ in effusion smear preparation correlates with that of underlying malignant pleural mesothelioma tissue. Cancer Sci 2015;106:1635-41.

56 Walters J, Maskell NA. Biopsy techniques for the diagnosis of mesothelioma. Recent Results Cancer Res 2011;189:45-55.

57 Segal A, Sterrett GF, Frost FA, et al. A diagnosis of malignant pleural mesothelioma can be made by effusion cytology: results of a 20 year audit. Pathology 2013:45:44-8

58 Cui $A$, Jin XG, Zhai K, et al. Diagnostic values of soluble mesothelin-related peptides for malignant pleural mesothelioma: updated meta-analysis. BMJ Open 2014:4:e004145

59 Hu ZD, Liu XF, Liu XC, et al. Diagnostic accuracy of osteopontin for malignant pleural mesothelioma: a systematic review and meta-analysis. Clin Chim Acta 2014;433:44-8.

60 Lin $\mathrm{H}$, Shen YC, Long HY, et al. Performance of osteopontin in the diagnosis of malignant pleural mesothelioma: a meta-analysis. Int J Clin Exp Med 2014;7:1289-96.

61 Kirschner MB, Pulford E, Hoda MA, et al. Fibulin-3 levels in malignant pleural mesothelioma are associated with prognosis but not diagnosis. $\mathrm{Br} J$ Cancer 2015;113:963-9.

62 Creaney J, Francis RJ, Dick IM, et al. Serum soluble mesothelin concentrations in malignant pleural mesothelioma: relationship to tumor volume, clinical stage and changes in tumor burden. Clin Cancer Res 2011;17:1181-9.
63 Creaney J, Dick IM, Segal A, et al. Pleural effusion hyaluronic acid as a prognostic marker in pleural malignant mesothelioma. Lung Cancer 2013;82:491-8.

64 Franko A, Dolzan V, Kovac V, et al. Soluble mesothelin-related peptides levels in patients with malignant mesothelioma. Dis Markers 2012:32:123-31.

65 Ghanim B, Hoda MA, Klikovits T, et al. Circulating fibrinogen is a prognostic and predictive biomarker in malignant pleural mesothelioma. Br J Cancer 2014;110:984-90.

66 Grigoriu BD, Chahine B, Vachani A, et al. Kinetics of soluble mesothelin in patients with malignant pleural mesothelioma during treatment. Am J Respir Crit Care Med 2009;179:950-4.

67 Hirayama N, Tabata C, Tabata R, et al. Pleural effusion VEGF levels as a prognostic factor of malignant pleural mesothelioma. Respir Med 2011;105:137-42.

68 Hollevoet K, Nackaerts K, Gosselin R, et al. Soluble mesothelin, megakaryocyte potentiating factor, and osteopontin as markers of patient response and outcome in mesothelioma. J Thorac Oncol 2011:6:1930-7.

69 Kao SC, Klebe S, Henderson DW, et al. Low calretinin expression and high neutrophil-to-lymphocyte ratio are poor prognostic factors in patients with malignant mesothelioma undergoing extrapleural pneumonectomy. J Thorac Oncol 2011;6:1923-9.

70 Kao SC, Harvie R, Paturi F, et al. The predictive role of serum VEGF in an advanced malignant mesothelioma patient cohort treated with thalidomide alone or combined with cisplatin/gemcitabine. Lung Cancer 2012;75:248-54.

71 Pinato DJ, Mauri FA, Ramakrishnan R, et al. Inflammation-based prognostic indices in malignant pleural mesothelioma. J Thorac Oncol 2012;7:587-94.

72 Thylén A, Hjerpe A, Martensson G. Hyaluronan content in pleural fluid as a prognostic factor in patients with malignant pleural mesothelioma. Cancer 2001;92:1224-30.

73 Wheatley-Price P, Yang B, Patsios D, et al. Soluble mesothelin-related Peptide and osteopontin as markers of response in malignant mesothelioma. J Clin Oncol 2010:28:3316-22.

74 Zhang Y, He J, Zhang F, et al. SMO expression level correlates with overall survival in patients with malignant pleural mesothelioma. J Exp Clin Cancer Res 2013;32:7.

75 Hooper CE, Lyburn ID, Searle J, et al. The South West Area Mesothelioma and Pemetrexed trial: a multicentre prospective observational study evaluating novel markers of chemotherapy response and prognostication. $\mathrm{Br} J$ Cancer 2015; 112:1175-82.

76 Hoda MA, Dong Y, Rozsas A, et al. Circulating activin A is a novel prognostic biomarker in malignant pleural mesothelioma - A multi-institutional study. Eur J Cancer 2016:63:64-73.

77 Park EK, Sandrini A, Yates DH, et al. Soluble mesothelin-related protein in an asbestos-exposed population: the dust diseases board cohort study. Am J Respir Crit Care Med 2008:178:832-7.

78 Filiberti R, Marroni P, Spigno F, et al. Is soluble mesothelin-related protein an upfront predictive marker of pleural mesothelioma? A prospective study on Italian workers exposed to asbestos. Oncology 2014;86:33-43.

79 Bayram M, Dongel I, Akbaș A, et al. Serum biomarkers in patients with mesothelioma and pleural plaques and healthy subjects exposed to naturally occurring asbestos. Lung 2014;192:197-203.

80 Gube M, Taeger D, Weber DG, et al. Performance of biomarkers SMRP, CA125, and CYFRA 21-1 as potential tumor markers for malignant mesothelioma and lung cancer in a cohort of workers formerly exposed to asbestos. Arch Toxicol 2011;85:185-92.

81 Rodríguez Portal JA, Rodríguez Becerra E, Rodríguez Rodríguez D, et al. Serum levels of soluble mesothelin-related peptides in malignant and nonmalignant asbestosrelated pleural disease: relation with past asbestos exposure. Cancer Epidemiol Biomarkers Prev 2009;18:646-50.

82 Bottomley A, Coens C, Efficace F, et al. Symptoms and patient-reported well-being: do they predict survival in malignant pleural mesothelioma? A prognostic facto analysis of EORTC-NCIC 08983: randomized phase III study of cisplatin with or without raltitrexed in patients with malignant pleural mesothelioma. J Clin Oncol 2007:25:5770-6.

83 Curran D, Sahmoud T, Therasse $\mathrm{P}$, et al. Prognostic factors in patients with pleural mesothelioma: the European Organization for Research and Treatment of Cance experience. J Clin Oncol 1998;16:145-52.

84 Fennell DA, Parmar A, Shamash J, et al. Statistical validation of the EORTC prognostic model for malignant pleural mesothelioma based on three consecutive phase II trials. J Clin Oncol 2005:23:184-9.

85 Meniawy TM, Creaney J, Lake RA, et al. Existing models, but not neutrophilto-lymphocyte ratio, are prognostic in malignant mesothelioma. Br J Cancer 2013; 109:1813-20.

86 Edwards JG, Abrams KR, Leverment JN, et al. Prognostic factors for malignant mesothelioma in 142 patients: validation of CALGB and EORTC prognostic scoring systems. Thorax 2000;55:731-5.

87 Herndon JE, Green MR, Chahinian AP, et al. Factors predictive of survival among 337 patients with mesothelioma treated between 1984 and 1994 by the Cancer and Leukemia Group B. Chest 1998;113:723-31. 
88 Clive AO, Kahan BC, Hooper CE, et al. Predicting survival in malignant pleural effusion: development and validation of the LENT prognostic score. Thorax 2014;69:1098-104.

89 Brims FJ, Meniawy TM, Duffus I, et al. A Novel Clinical Prediction Model for Prognosis in Malignant Pleural Mesothelioma Using Decision Tree Analysis. J Thorac Oncol 2016;11:573-82.

90 Gemba K, Fujimoto N, Aoe K, et al. Treatment and survival analyses of malignant mesothelioma in Japan. Acta Oncol 2013:52:803-8.

91 Milano MT, Zhang H. Malignant pleural mesothelioma: a population-based study of survival. J Thorac Oncol 2010;5:1841-8.

92 Taioli E, Wolf AS, Camacho-Rivera M, et al. Women with malignant pleural mesothelioma have a threefold better survival rate than men. Ann Thorac Surg 2014;98:1020-4.

93 Rintoul RC, Ritchie AJ, Edwards JG, et al. Efficacy and cost of video-assisted thoracoscopic partial pleurectomy versus talc pleurodesis in patients with malignant pleural mesothelioma (MesoVATS): an open-label, randomised, controlled trial. Lancet 2014:384:1118-27.

94 Davies HE, Mishra EK, Kahan BC, et al. Effect of an indwelling pleural catheter vs chest tube and talc pleurodesis for relieving dyspnea in patients with malignant pleural effusion: the TIME2 randomized controlled trial. JAMA 2012;307:2383-9.

95 Fysh ET, Tan SK, Read CA, et al. Pleurodesis outcome in malignant pleural mesothelioma. Thorax 2013;68:594-6.

96 Bielsa S, Hernández P, Rodriguez-Panadero F, et al. Tumor type influences the effectiveness of pleurodesis in malignant effusions. Lung 2011;189:151-5.

97 Aelony Y, Yao JF. Prolonged survival after talc poudrage for malignant pleural mesothelioma: case series. Respirology 2005;10:649-55.

98 Brancatisano RP, Joseph MG, McCaughan BC. Pleurectomy for mesothelioma. Med J Aust 1991;154:455-60.

99 Barbetakis N, Asteriou C, Papadopoulou F, et al. Early and late morbidity and mortality and life expectancy following thoracoscopic talc insufflation for control of malignant pleural effusions: a review of 400 cases. J Cardiothorac Surg 2010;5:27.

100 Basso SM, Mazza F, Marzano B, et al. Improved quality of life in patients with malignant pleural effusion following videoassisted thoracoscopic talc pleurodesis. Preliminary results. Anticancer Res 2012;32:5131-4.

101 Medford AR, Agrawal S, Free CM, et al. A local anaesthetic video-assisted thoracoscopy service: prospective performance analysis in a UK tertiary respiratory centre. Lung Cancer 2009;66:355-8.

102 Rice $\mathrm{D}$, Rusch V, Pass $\mathrm{H}$, et al. Recommendations for uniform definitions of surgical techniques for malignant pleural mesothelioma: a consensus report of the international association for the study of lung cancer international staging committee and the international mesothelioma interest group. J Thorac Oncol 2011:6:1304-12.

103 Treasure T, Lang-Lazdunski L, Waller D, et al. Extra-pleural pneumonectomy versus no extra-pleural pneumonectomy for patients with malignant pleural mesothelioma: clinical outcomes of the Mesothelioma and Radical Surgery (MARS) randomised feasibility study. Lancet Oncol 2011:12:763-72.

104 Mollberg NM, Vigneswaran Y, Kindler HL, et al. Quality of life after radical pleurectomy decortication for malignant pleural mesothelioma. Ann Thorac Surg 2012;94:1086-92.

105 Bölükbas S, Eberlein M, Schirren J. Prospective study on functional results after lung sparing radical pleurectomy in the management of malignant pleural mesothelioma. J Thorac Oncol 2012;7:900-5.

106 Burkholder D, Hadi D, Kunnavakkam R, et al. Effects of extended pleurectomy and decortication on quality of life and pulmonary function in patients with malignant pleural mesothelioma. Ann Thorac Surg 2015;99:1775-80.

107 Ploenes T, Osei-Agyemang T, Krohn A, et al. Changes in lung function after surgery for mesothelioma. Asian Cardiovasc Thorac Ann 2013:21:48-55.

108 Cao CQ, Yan TD, Bannon PG, et al. A systematic review of extrapleural pneumonectomy for malignant pleural mesothelioma. J Thorac Oncol 2010;5:1692-703

109 Cao C, Tian D, Manganas C, et al. Systematic review of trimodality therapy for patients with malignant pleural mesothelioma. Ann Cardiothorac Surg 2012;1:428-37.

110 Cao C, Tian DH, Pataky KA, et al. Systematic review of pleurectomy in the treatment of malignant pleural mesothelioma. Lung Cancer 2013;81:319-27.

111 Cao C, Tian D, Park J, et al. A systematic review and meta-analysis of surgical treatments for malignant pleural mesothelioma. Lung Cancer 2014;83:240-5.

112 Lim E. A feasibility study comparing (extended) pleurectomy decortication versus no pleurectomy decortication in the multimodality management of patients with malignant pleural mesothelioma: the MARS 2 study. Lung Cancer 2016;91((Suppl 1: S71)):S71.

113 Treasure T, Utley M, O'Byrne K. MARS: a sense of perspective and an inconvenient truth. J Thorac Oncol 2013;8:e48-e49.

114 Taioli E, Wolf AS, Flores RM. Meta-analysis of survival after pleurectomy decortication versus extrapleural pneumonectomy in mesothelioma. Ann Thorac Surg 2015;99:472-80
115 Vogelzang NJ, Rusthoven JJ, Symanowski J, et al. Phase III study of pemetrexed in combination with cisplatin versus cisplatin alone in patients with malignant pleural mesothelioma. J Clin Oncol 2003;21:2636-44.

116 van Meerbeeck JP, Gaafar R, Manegold C, et al. Randomized phase III study of cisplatin with or without raltitrexed in patients with malignant pleural mesothelioma: an intergroup study of the European Organisation for Research and Treatment of Cancer Lung Cancer Group and the National Cancer Institute of Canada. J Clin Oncol 2005:23:6881-9.

117 Muers MF, Stephens RJ, Fisher P, et al. Active symptom control with or without chemotherapy in the treatment of patients with malignant pleural mesothelioma (MS01): a multicentre randomised trial. Lancet 2008;371:1685-94.

118 Mordant P, Loriot Y, Soria JC, et al. MVP and vinorelbine for malignant pleural mesothelioma. Lancet 2008:372:629.

119 Zalcman G, Mazieres J, Margery J, et al. Bevacizumab 15mg/kg plus cisplatinpemetrexed (CP) triplet versus CP doublet in Malignant Pleural Mesothelioma (MPM): Results of the IFCT-GFPC-0701 MAPS randomized phase 3 trial. J of Clinical Oncology 2015:33(15 SUPPL. 1):7500.

120 Santoro A, O'Brien ME, Stahel RA, et al. Pemetrexed plus cisplatin or pemetrexed plus carboplatin for chemonaive patients with malignant pleural mesothelioma: results of the International Expanded Access Program. J Thorac Oncol 2008;3:756-63.

121 Buikhuisen WA, Hiddinga BI, Baas $\mathrm{P}$, et al. Second line therapy in malignant pleural mesothelioma: A systematic review. Lung Cancer 2015:89:223-31.

122 Jassem J, Ramlau R, Santoro A, et al. Phase III trial of pemetrexed plus best supportive care compared with best supportive care in previously treated patients with advanced malignant pleural mesothelioma. J Clin Oncol 2008;26:1698-704.

123 Krug LM, Kindler HL, Calvert H, et al. Vorinostat in patients with advanced malignant pleural mesothelioma who have progressed on previous chemotherapy (VANTAGE-014): a phase 3, double-blind, randomised, placebo-controlled trial. Lancet Oncol 2015;16:447-56.

124 Thapa B, Salcedo A, Lin X, et al. The Immune Microenvironment, Genome-wide Copy Number Aberrations, and Survival in Mesothelioma. J Thorac Oncol 2017:12:850-9.

125 Alley EW, Lopez J, Santoro A, et al. Clinical safety and activity of pembrolizumab in patients with malignant pleural mesothelioma (KEYNOTE-028): preliminary results from a non-randomised, open-label, phase 1b trial. Lancet Oncol 2017:18:623-30.

126 Boutin C, Rey F, Viallat JR. Prevention of malignant seeding after invasive diagnostic procedures in patients with pleural mesothelioma. A randomized trial of local radiotherapy. Chest 1995; 108:754-8.

127 Bydder S, Phillips M, Joseph DJ, et al. A randomised trial of single-dose radiotherapy to prevent procedure tract metastasis by malignant mesothelioma. $\mathrm{Br} J$ Cancer 2004;91:9-10

128 O'Rourke N, Garcia JC, Paul J, et al. A randomised controlled trial of intervention site radiotherapy in malignant pleural mesothelioma. Radiother Oncol 2007;84:18-22.

129 Clive A0, Taylor H, Dobson L, et al. Prophylactic radiotherapy for the prevention of procedure-tract metastases after surgical and large-bore pleural procedures in malignant pleural mesothelioma (SMART): a multicentre, open-label, phase 3 , randomised controlled trial. Lancet Oncol 2016;17:1094-104.

130 Lee C, Bayman N, Swindell R, et al. Prophylactic radiotherapy to intervention sites in mesothelioma: a systematic review and survey of UK practice. Lung Cancer 2009:66:150-6.

131 Bayman N, Ardron D, Ashcroft L, et al. Protocol for PIT: a phase III trial of prophylactic irradiation of tracts in patients with malignant pleural mesothelioma following invasive chest wall intervention. BMJ Open 2016;6:e010589.

132 de Perrot M, Feld R, Leighl NB, et al. Accelerated hemithoracic radiation followed by extrapleural pneumonectomy for malignant pleural mesothelioma. J Thorac Cardiovasc Surg 2016;151:468-75.

133 Lindén CJ, Mercke C, Albrechtsson U, et al. Effect of hemithorax irradiation alone or combined with doxorubicin and cyclophosphamide in 47 pleural mesotheliomas: a nonrandomized phase II study. Eur Respir J 1996:9:2565-72.

134 Rimner A, Spratt DE, Zauderer MG, et al. Failure patterns after hemithoracic pleural intensity modulated radiation therapy for malignant pleural mesothelioma. Int $J$ Radiat Oncol Biol Phys 2014;90:394-401.

135 Allen AM, Czerminska M, Jänne PA, et al. Fatal pneumonitis associated with intensity-modulated radiation therapy for mesothelioma. Int I Radiat Oncol Biol Phys 2006;65:640-5

136 Allen AM, Den R, Wong JS, et al. Influence of radiotherapy technique and dose on patterns of failure for mesothelioma patients after extrapleural pneumonectomy. Int J Radiat Oncol Biol Phys 2007;68:1366-74.

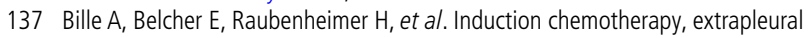
pneumonectomy, and adjuvant radiotherapy for malignant pleural mesothelioma: experience of Guy's and St Thomas' hospitals. Gen Thorac Cardiovasc Surg 2012:60:289-96.

138 Bölükbas S, Manegold C, Eberlein M, et al. Survival after trimodality therapy for malignant pleural mesothelioma: Radical Pleurectomy, chemotherapy with Cisplatin/ Pemetrexed and radiotherapy. Lung Cancer 2011;71:75-81.

139 Flores RM, Krug LM, Rosenzweig KE, et al. Induction chemotherapy, extrapleura pneumonectomy, and postoperative high-dose radiotherapy for locally advanced malignant pleural mesothelioma: a phase II trial. J Thorac Oncol 2006;1:289-95. 
140 Kristensen CA, Nøttrup TJ, Berthelsen AK, et al. Pulmonary toxicity following IMRT after extrapleural pneumonectomy for malignant pleural mesothelioma. Radiother Oncol 2009;92:96-9.

141 Lucchi M, Chella A, Melfi F, et al. Four-modality therapy in malignant pleural mesothelioma: a phase II study. J Thorac Oncol 2007;2:237-42.

142 Minatel E, Trovo M, Polesel J, et al. Tomotherapy after pleurectomy/decortication or biopsy for malignant pleural mesothelioma allows the delivery of high dose of radiation in patients with intact lung. J Thorac Oncol 2012;7:1862-6.

143 Minatel E, Trovo M, Polesel J, et al. Radical pleurectomy/decortication followed by high dose of radiation therapy for malignant pleural mesothelioma. Final results with long-term follow-up. Lung Cancer 2014;83:78-82.

144 Rice DC, Smythe WR, Liao Z, et al. Dose-dependent pulmonary toxicity after postoperative intensity-modulated radiotherapy for malignant pleural mesothelioma. Int J Radiat Oncol Biol Phys 2007;69:350-7.

145 Pagan V, Ceron L, Paccagnella A, et al. 5-year prospective results of trimodality treatment for malignant pleural mesothelioma. J Cardiovasc Surg 2006;47:595-601.

146 Rusch VW, Rosenzweig K, Venkatraman E, et al. A phase II trial of surgical resection and adjuvant high-dose hemithoracic radiation for malignant pleural mesothelioma. J Thorac Cardiovasc Surg 2001;122:788-95.

147 Tonoli S, Vitali P, Scotti V, et al. Adjuvant radiotherapy after extrapleural pneumonectomy for mesothelioma. Prospective analysis of a multi-institutional series. Radiother Oncol 2011;101:311-5.

148 Van Schil PE, Baas P, Gaafar R, et al. Trimodality therapy for malignant pleural mesothelioma: results from an EORTC phase II multicentre trial. Eur Respir J 2010;36:1362-9.

149 Weder W, Stahel RA, Bernhard J, et al. Multicenter trial of neo-adjuvant chemotherapy followed by extrapleural pneumonectomy in malignant pleural mesothelioma. Ann Oncol 2007;18:1196-202.

150 Stahel RA, Riesterer 0, Xyrafas A, et al. Neoadjuvant chemotherapy and extrapleura pneumonectomy of malignant pleural mesothelioma with or without hemithoracic radiotherapy (SAKK 17/04): a randomised, international, multicentre phase 2 trial. Lancet Oncol 2015;16:1651-8.

151 Rimner A, Zauderer MG, Gomez DR, et al. Phase II Study of Hemithoracic Intensity-Modulated Pleural Radiation Therapy (IMPRINT) As Part of Lung-Sparing Multimodality Therapy in Patients With Malignant Pleural Mesothelioma. J Clin Oncol 2016:34:2761-8

152 Bissett D, Macbeth FR, Cram I. The role of palliative radiotherapy in malignant mesothelioma. Clin Oncol 1991:3:315-7.

153 MacLeod N, Chalmers A, O'Rourke N, et al. Is Radiotherapy Useful for Treating Pain in Mesothelioma?: A Phase II Trial. J Thorac Oncol 2015;10:944-50.

154 Davis SR, Tan L, Ball DL. Radiotherapy in the treatment of malignant mesothelioma of the pleura, with special reference to its use in palliation. Australas Radiol 1994;38:212-4.

155 de Graaf-Strukowska L, van der Zee J, van Putten W, et al. Factors influencing the outcome of radiotherapy in malignant mesothelioma of the pleura--a single-institution experience with 189 patients. Int J Radiat Oncol Biol Phys 1999:43:511-6.

156 Jenkins P, Milliner R, Salmon C. Re-evaluating the role of palliative radiotherapy in malignant pleural mesothelioma. Eur J Cancer 2011:47:2143-9.

157 Chapman E, Berenstein EG, Dieguez M, et al. Radiotherapy for malignant pleural mesothelioma. Cochrane Database Syst Rev 2006;3:CD003880.

158 Macleod N, Price A, O'Rourke N, et al. Radiotherapy for the treatment of pain in malignant pleural mesothelioma: a systematic review. Lung Cancer 2014:83:133-8

159 Jackson MB, Pounder D, Price C, et al. Percutaneous cervical cordotomy for the control of pain in patients with pleural mesothelioma. Thorax 1999;54:238-41.

160 McNair AG, Choh CT, Metcalfe C, et al. Maximising recruitment into randomised controlled trials: the role of multidisciplinary cancer teams. Eur J Cancer 2008:44:2623-6.

161 Taylor C, Shewbridge A, Harris J, et al. Benefits of multidisciplinary teamwork in the management of breast cancer. Breast Cancer 2013;5:79-85.

162 Taplin SH, Weaver S, Salas E, et al. Reviewing cancer care team effectiveness. J Oncol Pract 2015;11:239-46.

163 Munro AJ, Swartzman S. What is a virtual multidisciplinary team (vMDT)? Br J Cancer 2013; 108:2433-41.

164 Bibby AC, Williams K, Smith S, et al. What is the role of a specialist regional mesothelioma multidisciplinary team meeting? A service evaluation of one tertiary referral centre in the UK. BMJ Open 2016:6:e012092.

165 NICE. Lung cancer: diagnosis and management, 2011. CG121.

166 Granieri A, Tamburello S, Tamburello A, et al. Quality of life and personality traits in patients with malignant pleural mesothelioma and their first-degree caregivers. Neuropsychiatr Dis Treat 2013;9:1193-202.

167 Arber A, Spencer L. 'It's all bad news': the first 3 months following a diagnosis of malignant pleural mesothelioma. Psychooncology 2013;22:1528-33.

168 Clayson H, Seymour J, Noble B. Mesothelioma from the patient's perspective. Hematol Oncol Clin North Am 2005;19:1175-90.
169 Ball H, Moore S, Leary A. A systematic literature review comparing the psychological care needs of patients with mesothelioma and advanced lung cancer. Eur J Oncol Nurs 2016;25:62-7

170 Moore S, Teehan C, Cornwall A, et al. 'Hands of Time': the experience of establishing a support group for people affected by mesothelioma. Eur J Cancer Care 2008:17:585-92.

171 Chamming's S, Clin B, Brochard P, et al. Compensation of pleural mesothelioma in France: data from the French National Mesothelioma Surveillance Programme. Am J Ind Med 2013;56:146-54.

172 Cree MW, Lalji M, Jiang B, et al. Under-reporting of compensable mesothelioma in Alberta. Am J Ind Med 2009;52:526-33.

173 Kuschner WG, Varma R, Flores R, et al. Missed opportunities to counsel patients with malignant pleural mesothelioma about causation and potential compensation. Am J Med Sci 2012;343:206-9.

174 Ak G, Metintas M, Metintas S, et al. Three-dimensional evaluation of chemotherapy response in malignant pleural mesothelioma. Eur J Radiol 2010;74:130-5.

175 Armato SG, Nowak AK, Francis RJ, et al. Observer variability in mesothelioma tumor thickness measurements: defining minimally measurable lesions. J Thorac Oncol 2014;9:1187-94.

176 Byrne MJ, Nowak AK. Modified RECIST criteria for assessment of response in malignant pleural mesothelioma. Annals of Oncology 2004;15:257-60.

177 Ceresoli GL, Chiti A, Zucali PA, et al. Early response evaluation in malignant pleural mesothelioma by positron emission tomography with [18F]fluorodeoxyglucose. J Clin Oncol 2006:24:4587-93.

178 Frauenfelder T, Tutic M, Weder W, et al. Volumetry: an alternative to assess therapy response for malignant pleural mesothelioma? Eur Respir J 2011:38:162-8.

179 Hilmi Ozden SM. Mustaffer Metintas Relationship between tumour size of MPM and its response to chemotherapy. Journal of Health Science 2007;53:23-33.

180 Nowak AK. CT, RECIST, and malignant pleural mesothelioma. Lung Cancer 2005;49(Suppl 1):S37-S40.

181 Plathow C, Klopp M, Thieke C, et al. Therapy response in malignant pleural mesothelioma-role of MRI using RECIST, modified RECIST and volumetric approaches in comparison with CT. Eur Radiol 2008;18:1635-43.

182 van Klaveren RJ, Aerts JG, de Bruin H, et al. Inadequacy of the RECIST criteria for response evaluation in patients with malignant pleural mesothelioma. Lung Cancer 2004; $43: 63-9$

183 Byrne MJ, Nowak AK. Modified RECIST criteria for assessment of response in malignant pleural mesothelioma. Ann Oncol 2004;15:257-60.

184 Plathow C, Klopp M, Thieke C, et al. Therapy response in malignant pleural mesothelioma-role of MRI using RECIST, modified RECIST and volumetric approaches in comparison with CT[Erratum appears in Eur Radiol. 2010 Apr;20(4):1023]. Eur Radiol 2008:18:1635-43.

185 NICE. Suspected cancer: recognition and referral, 2015. NG 12.

186 Carella R, Deleonardi G, D'Errico A, et al. Immunohistochemical panels for differentiating epithelial malignant mesothelioma from lung adenocarcinoma: a study with logistic regression analysis. Am J Surg Pathol 2001;25:43-50.

187 Klebe S, Nurminen M, Leigh J, et al. Diagnosis of epithelial mesothelioma using tree-based regression analysis and a minimal panel of antibodies. Pathology 2009; 41:140-8.

188 Lucas DR, Pass HI, Madan SK, et al. Sarcomatoid mesothelioma and its histological mimics: a comparative immunohistochemical study. Histopathology 2003;42:270-9.

189 Ordóñez NG. Mesothelioma with signet-ring cell features: report of 23 cases. Mod Pathol 2013;26:370-84

190 Brockstedt U, Gulyas M, Dobra K, et al. An optimized battery of eight antibodies that can distinguish most cases of epithelial mesothelioma from adenocarcinoma. Am J Clin Pathol 2000;114:203-9.

191 Comin CE, Dini S, Novelli L, et al. h-Caldesmon, a useful positive marker in the diagnosis of pleural malignant mesothelioma, epithelioid type. Am J Surg Pathol 2006;30:463-9.

192 Comin CE, Novelli L, Boddi V, et al. Calretinin, thrombomodulin, CEA, and CD15: a useful combination of immunohistochemical markers for differentiating pleural epithelial mesothelioma from peripheral pulmonary adenocarcinoma. Hum Pathol 2001:32:529-36.

193 Cury PM, Butcher DN, Corrin B, et al. The use of histological and immunohistochemical markers to distinguish pleural malignant mesothelioma and in situ mesothelioma from reactive mesothelial hyperplasia and reactive pleural fibrosis. J Pathol 1999;189:251-7.

194 Cury PM, Butcher DN, Fisher C, et al. Value of the mesothelium-associated antibodies thrombomodulin, cytokeratin 5/6, calretinin, and CD44H in distinguishing epithelioid pleural mesothelioma from adenocarcinoma metastatic to the pleura. Mod Pathol 2000;13:107-12.

195 Takeshima Y, Amatya VJ, Kushitani K, et al. Value of immunohistochemistry in the differential diagnosis of pleural sarcomatoid mesothelioma from lung sarcomatoid carcinoma. Histopathology 2009;54:667-76.

196 Roberts F, Harper CM, Downie I, et al. Immunohistochemical analysis still has a limited role in the diagnosis of malignant mesothelioma. A study of thirteen antibodies. Am J Clin Pathol 2001;116:253-62. 
197 Mimura T, Ito A, Sakuma T, et al. Novel marker D2-40, combined with calretinin, CEA, and TTF-1: an optimal set of immunodiagnostic markers for pleural mesothelioma. Cancer 2007;109:933-8.

198 Miettinen M, Sarlomo-Rikala M. Expression of calretinin, thrombomodulin, keratin 5, and mesothelin in lung carcinomas of different types: an immunohistochemical analysis of 596 tumors in comparison with epithelioid mesotheliomas of the pleura. Am J Surg Pathol 2003;27:150-8.

199 Leers MP, Aarts MM, Theunissen PH. E-cadherin and calretinin: a useful combination of immunochemical markers for differentiation between mesothelioma and metastatic adenocarcinoma. Histopathology 1998;32:209-16.

200 Klebe S, Brownlee NA, Mahar A, et al. Sarcomatoid mesothelioma: a clinicalpathologic correlation of 326 cases. Mod Pathol 2010:23:470-9.

201 Gotzos V, Vogt P, Celio MR. The calcium binding protein calretinin is a selective marker for malignant pleural mesotheliomas of the epithelial type. Pathol Res Pract 1996;192:137-47.

202 Attanoos RL, Goddard H, Gibbs AR. Mesothelioma-binding antibodies: thrombomodulin, OV 632 and HBME-1 and their use in the diagnosis of malignant mesothelioma. Histopathology 1996;29:209-15.

203 Brown RW, Clark GM, Tandon AK, et al. Multiple-marker immunohistochemical phenotypes distinguishing malignant pleural mesothelioma from pulmonary adenocarcinoma. Hum Pathol 1993;24:347-54.

204 Collins CL, Ordonez NG, Schaefer R, et al. Thrombomodulin expression in malignant pleural mesothelioma and pulmonary adenocarcinoma. Am J Pathol 1992:141:827-33.

205 Dejmek A, Brockstedt U, Hjerpe A. Optimization of a battery using nine immunocytochemical variables for distinguishing between epithelial mesothelioma and adenocarcinoma. APMIS 1997; 105:889-94.

206 Dejmek A, Hjerpe A. The combination of CEA, EMA, and BerEp4 and hyaluronan analysis specifically identifies $79 \%$ of all histologically verified mesotheliomas causing an effusion. Diagn Cytopathol 2005;32:160-6.

207 Fetsch PA, Abati A, Hijazi YM. Utility of the antibodies CA 19-9, HBME-1, and thrombomodulin in the diagnosis of malignant mesothelioma and adenocarcinoma in cytology. Cancer 1998;84:101-8.

208 Ordóñez NG. The value of antibodies 44-3A6, SM3, HBME-1, and thrombomodulin in differentiating epithelial pleural mesothelioma from lung adenocarcinoma: a comparative study with other commonly used antibodies. Am J Surg Pathol 1997:21:1399-408

209 Ordóñez NG. Value of thrombomodulin immunostaining in the diagnosis of mesothelioma. Histopathology 1997:31:25-30.

210 Kennedy AD, King G, Kerr KM. HBME-1 and antithrombomodulin in the differential diagnosis of malignant mesothelioma of pleura. J Clin Pathol 1997:50:859-62.

211 Clover J, Oates J, Edwards C. Anti-cytokeratin 5/6: a positive marker for epithelioid mesothelioma. Histopathology 1997:31:140-3.

212 González-Lois C, Ballestín C, Sotelo MT, et al. Combined use of novel epithelial (MOC-31) and mesothelial (HBME-1) immunohistochemical markers for optimal first line diagnostic distinction between mesothelioma and metastatic carcinoma in pleura. Histopathology 2001;38:528-34

213 Delahaye M, van der Ham F, van der Kwast TH. Complementary value of five carcinoma markers for the diagnosis of malignant mesothelioma, adenocarcinoma metastasis, and reactive mesothelium in serous effusions. Diagn Cytopathol 1997; 17:115-20.

214 Garcia-Prats MD, Ballestin C, Sotelo T, et al. A comparative evaluation of immunohistochemical markers for the differential diagnosis of malignant pleural tumours. Histopathology 1998;32:462-72.

215 Dejmek A, Hjerpe A. Reactivity of six antibodies in effusions of mesothelioma adenocarcinoma and mesotheliosis: stepwise logistic regression analysis. Cytopathology 2000;11:8-17.

216 Aerts JG, Delahaye M, van der Kwast TH, et al. The high post-test probability of a cytological examination renders further investigations to establish a diagnosis of epithelial malignant pleural mesothelioma redundant. Diagn Cytopathol 2006:34:523-7.

217 al-Saffar N, Hasleton PS, Vimentin HPS. Vimentin, carcinoembryonic antigen and keratin in the diagnosis of mesothelioma, adenocarcinoma and reactive pleural lesions. Eur Respir J 1990;3:997-1001.

218 Wick MR, Loy T, Mills SE, et al. Malignant epithelioid pleural mesothelioma versus peripheral pulmonary adenocarcinoma: a histochemical, ultrastructural, and immunohistologic study of 103 cases. Hum Pathol 1990;21:759-66.

219 Bakir K, Koçer NE, Deniz H, et al. TTF-1 and surfactant-B as co-adjuvants in the diagnosis of lung adenocarcinoma and pleural mesothelioma. Ann Diagn Pathol 2004;8:337-41.
220 Di Loreto C, Puglisi F, Di Lauro V, et al. TTF-1 protein expression in pleural malignant mesotheliomas and adenocarcinomas of the lung. Cancer Lett 1998:124:73-8.

221 Attanoos RL, Griffin A, Gibbs AR. The use of immunohistochemistry in distinguishing reactive from neoplastic mesothelium. A novel use for desmin and comparative evaluation with epithelial membrane antigen, p53, platelet-derived growth factorreceptor, P-glycoprotein and Bcl-2. Histopathology 2003;43:231-8.

222 Bateman AC, al-Talib RK, Newman T, et al. Immunohistochemical phenotype of malignant mesothelioma: predictive value of CA125 and HBME-1 expression. Histopathology 1997;30:49-56.

223 Ordóñez NG. Value of thyroid transcription factor-1, E-cadherin, BG8, WT1, and CD44S immunostaining in distinguishing epithelial pleural mesothelioma from pulmonary and nonpulmonary adenocarcinoma. Am J Surg Pathol 2000:24:598-606.

224 Cagle PT, Brown RW, Lebovitz RM. p53 immunostaining in the differentiation of reactive processes from malignancy in pleural biopsy specimens. Hum Pathol 1994; $25: 443-8$

225 Husain AN, Mirza MK, Gibbs A, et al. How useful is GLUT-1 in differentiating mesothelial hyperplasia and fibrosing pleuritis from epithelioid and sarcomatoid mesotheliomas? An international collaborative study. Lung Cancer 2014;83:324-8.

226 Kato Y, Tsuta K, Seki K, et al. Immunohistochemical detection of GLUT-1 can discriminate between reactive mesothelium and malignant mesothelioma. Mod Pathol 2007:20:215-20.

227 Kawamura K, Hiroshima K, Suzuki T, et al. CD90 is a diagnostic marker to differentiate between malignant pleural mesothelioma and lung carcinoma with immunohistochemistry. Am J Clin Pathol 2013;140:544-9.

228 Jo VY, Cibas ES, Pinkus GS. Claudin-4 immunohistochemistry is highly effective in distinguishing adenocarcinoma from malignant mesothelioma in effusion cytology. Cancer Cytopathol 2014;122:299-306.

229 Ordóñez NG. D2-40 and podoplanin are highly specific and sensitive immunohistochemical markers of epithelioid malignant mesothelioma. Hum Pathol 2005;36:372-80

230 Pass HI, Levin SM, Harbut MR, et al. Fibulin-3 as a blood and effusion biomarker for pleural mesothelioma. N Engl J Med 2012;367:1417-27.

231 Agha MA, El-Habashy MM, El-Shazly RA. Role of fibulin-3 in the diagnosis of malignant mesothelioma. Egypt J Chest Dis Tuberc 2014;63:99-105.

232 Elgazzar AeldinM, Embarak S, Refat AM, et al. Value of plasma and pleural effusion fibulin-3 levels in the diagnosis of malignant pleural mesothelioma effusions. Egypt $J$ Chest Dis Tuberc 2014;63:883-8.

233 Creaney J, Dick IM, Meniawy TM, et al. Comparison of fibulin-3 and mesothelin as markers in malignant mesothelioma. Thorax 2014;69:895-902.

234 Jennings AL, Davies AN, Higgins JP, et al. A systematic review of the use of opioids in the management of dyspnoea. Thorax 2002;57:939-44.

235 Abernethy AP, Currow DC, Frith P, et al. Randomised, double blind, placebo controlled crossover trial of sustained release morphine for the management of refractory dyspnoea. BMJ 2003;327:523-8

236 Bausewein C, Booth S, Gysels M, et al. Non-pharmacological interventions for breathlessness in advanced stages of malignant and non-malignant diseases. Cochrane Database Syst Rev 2008:2:CD005623.

237 Galbraith S, Fagan P, Perkins P, et al. Does the use of a handheld fan improve chronic dyspnea? A randomized, controlled, crossover trial. J Pain Symptom Manage 2010;39:831-8

238 Higginson IJ, Bausewein C, Reilly CC, et al. An integrated palliative and respiratory care service for patients with advanced disease and refractory breathlessness: a randomised controlled trial. Lancet Respir Med 2014;2:979-87.

239 Farquar M. Is a specialist breathlessness service more effective and cost-effective for patients with advanced cancer and their carers than standard care? BMC Med 2014;12:194.

240 Caraceni A, Hanks G, Kaasa S, et al. Use of opioid analgesics in the treatment of cancer pain: evidence-based recommendations from the EAPC. Lancet Oncol 2012;13:e58-e68.

241 NICE. Opiods in palliative care:safe and effective prescribing of strong opiods in palliative care of adults, 2012. CG 140

242 NICE. Neuropathic pain - pharmacological management, 2013. CG 173.

243 Finnerup NB, Attal N, Haroutounian S, et al. Pharmacotherapy for neuropathic pain in adults: a systematic review and meta-analysis. Lancet Neuro/ 2015;14:162-73.

244 Cramp F, Daniel J. Exercise for the management of cancer-related fatigue in adults. Cochrane database of systematic reviews (Online). Cochrane Database Syst Rev 2012:11:CD006145.

245 Ruiz Garcia V, López-Briz E, Carbonell Sanchis R, et al. Megestrol acetate for treatment of anorexia-cachexia syndrome. Cochrane Database Syst Rev 2013;9:CD004310 


\section{APPENDIX 1: LIST OF GUIDELINE GROUP MEMBERS}

Professor Nick Maskell, Respiratory Physician, Bristol.

Dr Ian Woolhouse, Respiratory Physician, Birmingham. Representing the Royal College of Physicians.

Dr Lesley Bishop, Respiratory Physician, Portsmouth.

Ms Liz Darlison, Respiratory Nurse Specialist, Leicester.

Dr Duneesha de Fonseka, Specialist Trainee, Bristol.

Dr Anthony Edey, Radiologist, Bristol. Representing the British Society of Thoracic Imaging and the Royal College of Radiologists.

Mr John Edwards, Thoracic Surgeon, Sheffield. Representing the Society of Cardiothoracic Surgeons and the British Thoracic Oncology Group.

Professor Corinne Faivre-Finn, Oncologist, Leicester. Representing the Royal College of Radiologists.

Professor Dean A Fennell, Oncologist, Leicester. Representing the Association of Cancer Physicians.

Dr Steve Holmes, General Practitioner. Representing Primary Care Respiratory Society UK.

Professor Keith Kerr, Histopathologist, Aberdeen. Representing Royal College of Pathologists.

Mr Apostolos Nakas, Thoracic Surgeon, Leicester. Representing Society of Cardiothoracic Surgeons.

Dr Tim Peel, Palliative and Respiratory Physician. Representing the Association for Palliative Medicine.

Professor Najib Rahman, Respiratory Physician, Oxford.

Dr Mark Slade, Respiratory Physician, Cambridge.

Dr Jeremy Steele, Oncologist, London. Representing the Association of Cancer Physicians.

Dr Selina Tsim, Specialty Trainee, Glasgow.

Contributors:

The lay representatives on the group were Dr Graham Abbott, Mr Paul Astle and Mr John Gillies.

Additional nursing contributors to the Guideline Development Group were Ms Sarah Smith (Mar 2015-Oct 2015) and Ms Gerry

Slade (until Mar 2015). 


\section{APPENDIX 2: PROGNOSTIC SCORES}

\section{The EORTC Prognostic Score}

The score is:

$\mathrm{EPS}=0.55$ (if $\mathrm{WBC}>8.3 \times 109 / \mathrm{L})+0.6$ (if $\mathrm{PS}=1$ or 2 ) +0.52 (if histological diagnosis probable or possible) +0.67 (if histology $=$ sarcomatoid $)+0.6$ (if male)

The patient has a good prognosis if EPS $<=1.27$ and a poor prognosis if EPS $>1.27$.

The Neutrophil-to-Lymphocyte ratio (NLR)

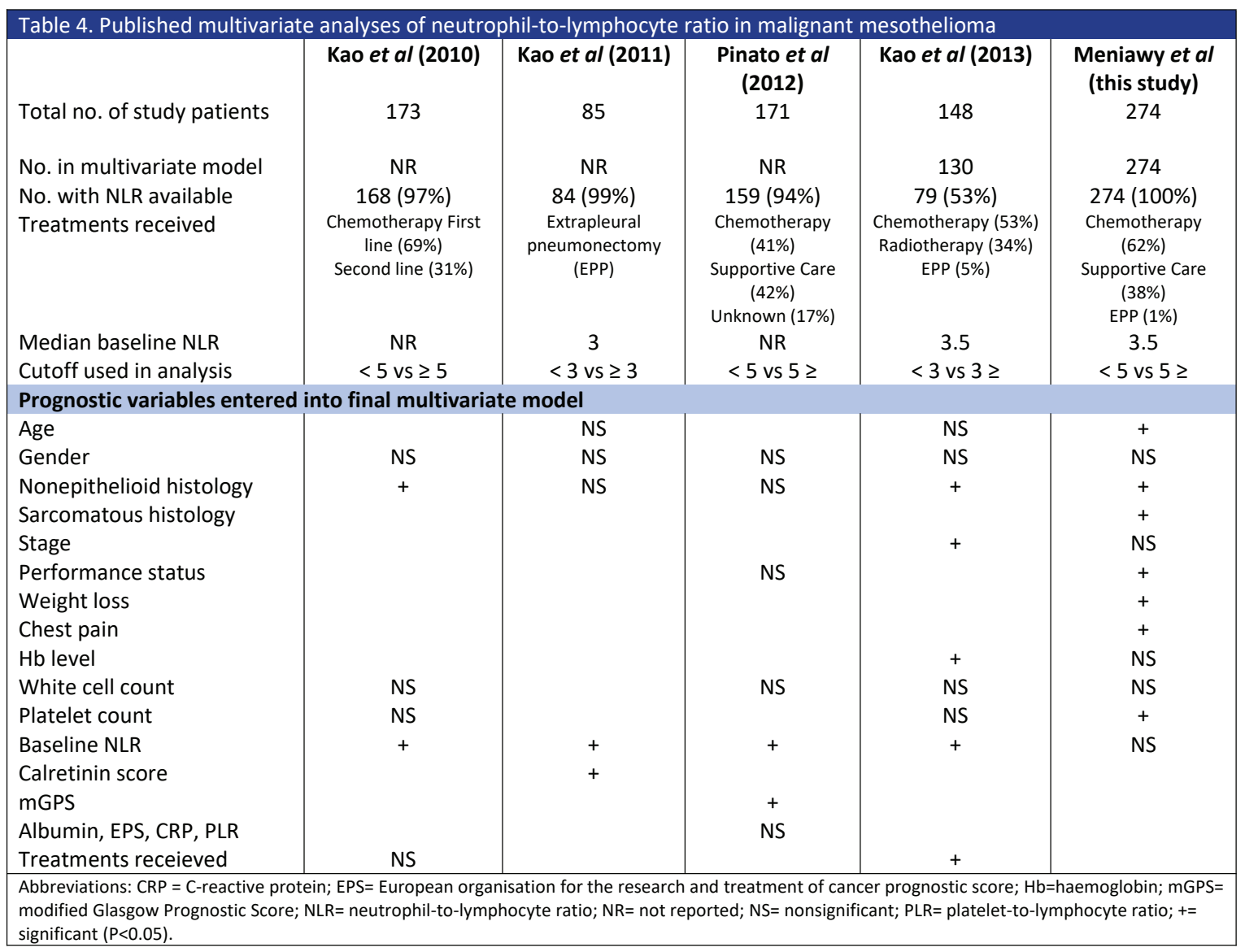




\section{BTS guideline}

\section{PROGNOSTIC MODEL USING DECISION TREE ANALYSIS}

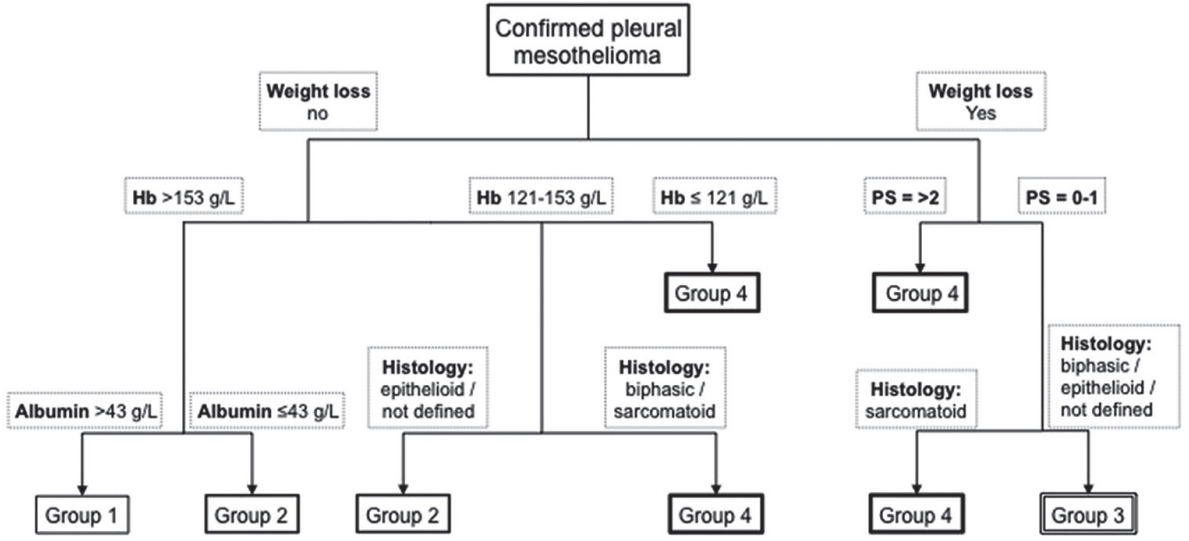

Reprinted with permission from Elsevier. From Brims FJ, Meniawy TM, Duffus I, et al. A Novel clinical prediction model for prognosis in Malignant Pleural Mesothelioma using decision tree analysis. J Thorac Oncol 2016;11(4):573-82.

\section{The LENT scoring system}

\begin{tabular}{|l|l|l|}
\hline \multirow{4}{*}{ Mnemonic } & Variable & Score \\
\hline \multirow{4}{*}{ E } & Pleural fluid LDH (IU/L) & \\
\cline { 2 - 3 } & $<1500$ & 0 \\
\cline { 2 - 3 } & $>1500$ & 1 \\
\hline \multirow{5}{*}{ N } & ECOG Performance Status & \\
\cline { 2 - 3 } & 0 & 0 \\
\cline { 2 - 3 } & 1 & 1 \\
\cline { 2 - 3 } & 2 & 2 \\
\cline { 2 - 3 } & $3-4$ & 3 \\
\hline \multirow{5}{*}{ T } & NLR & 0 \\
\cline { 2 - 3 } & $<9$ & 1 \\
\cline { 2 - 3 } & $>9$ & 2 \\
\hline & Tumour type & \\
\cline { 2 - 3 } & Low risk (mesothelioma, haematological malignancy) & 0 \\
\cline { 2 - 3 } & Moderate risk (breast, renal, gynaecological cancer) & 1 \\
\cline { 2 - 3 } & High risk (lung cancer, other tumour types) & \\
\hline
\end{tabular}

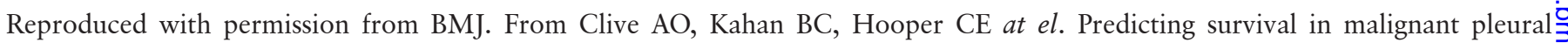
effusion: development and validation of the LENT prognostic score. Thorax 2014;69:1098-104. 